MATHEMATICS OF COMPUTATION

Volume 74, Number 251, Pages 1345-1367

S 0025-5718(04)01704-1

Article electronically published on September 10, 2004

\title{
NONINTERPOLATORY HERMITE SUBDIVISION SCHEMES
}

\author{
BIN HAN, THOMAS P.-Y. YU, AND YONGGANG XUE
}

\begin{abstract}
Bivariate interpolatory Hermite subdivision schemes have recently been applied to build free-form subdivision surfaces. It is well known to geometric modelling practitioners that interpolatory schemes typically lead to "unfair" surfaces - surfaces with unwanted wiggles or undulations - and noninterpolatory (a.k.a. approximating in the CAGD community) schemes are much preferred in geometric modelling applications. In this article, we introduce, analyze and construct noninterpolatory Hermite subdivision schemes, a class of vector subdivision schemes which can be applied to iteratively refine Hermite data in a not necessarily interpolatory fashion. We also study symmetry properties of such subdivision schemes which are crucial for application in free-form subdivision surfaces.

A key step in our mathematical analysis of Hermite type subdivision schemes is that we make use of the strong convergence theory of refinement equations to convert a prescribed geometric condition on the subdivision scheme - namely, the subdivision scheme is of Hermite type - to an algebraic condition on the subdivision mask. The latter algebraic condition can then be used in a computational framework to construct specific schemes.
\end{abstract}

\section{INTRODUCTION}

Subdivision algorithms are iterative methods for producing smooth curves and surfaces with a built-in multiresolution structure. They are now used in surface modelling in computer-aided geometric design. They are also intimately connected to wavelet bases and their associated fast filter bank algorithms. In the so-called functional/parametric, shift-invariant/regular setting, a subdivision scheme is a linear operator $S:=S_{\mathbf{a}, M}:\left[l\left(\mathbb{Z}^{s}\right)\right]^{1 \times m} \rightarrow\left[l\left(\mathbb{Z}^{s}\right)\right]^{1 \times m}$ of the form

$$
S v(\alpha)=\sum_{\beta \in \mathbb{Z}^{s}} v(\beta) \mathbf{a}(\alpha-M \beta),
$$

where $\mathbf{a} \in\left[l^{0}\left(\mathbb{Z}^{s}\right)\right]^{m \times m}$ is the mask of the subdivision scheme, $M$ is the dilation matrix and $m$ is the multiplicity of the subdivision scheme.

Received by the editor April 15, 2003 and, in revised form, December 10, 2003.

2000 Mathematics Subject Classification. Primary 41A05, 41A15, 41A63, 42C40, 65T60, $65 \mathrm{~F} 15$

Key words and phrases. Refinable function, vector refinability, subdivision scheme, shift invariant subspace, subdivision surface, spline.

The first author's research was supported in part by the Natural Sciences and Engineering Research Council of Canada (NSERC Canada) under grant G121210654.

The second author's research was supported in part by an NSF CAREER Award (CCR 9984501).

(C)2004 American Mathematical Society 
General vector subdivision schemes. Vector subdivision schemes and vector refinement equations are very related and had been extensively studied in the mathematics literature. Since free-form subdivision surfaces in geometric modelling is an application domain where scalar (i.e., $m=1$ ) subdivision schemes find major practical applications (see, e.g., 6, 1, 23, 9, 29, 22, 21, 26, 27]), one would expect that the extensive theory in vector subdivision schemes can be materialized into useful algorithms for modelling free-form surfaces, potentially with improved properties in specific situations. In implementing this proposal, the first fundamental difficulty we encounter occurs to be that the subdivision data generated by a general vector subdivision scheme has a rather unclear geometric meaning.

For any convergent scalar scheme $S$ one has, writing informally,

$$
S^{n} v(\alpha) \approx f\left(M^{-n} \alpha\right), \quad n \text { large. }
$$

This means that the scalar $S^{n} v(\alpha)$ "measures" the "approximate position" of a point on the limit curve (when $s=1$ ) or surface (when $s=2$ ). In a vector scheme, however, the different components in the vector $S^{n} v(\alpha)$ have, in general, no explicit "geometric meanings". To clarify as much as possible what we mean here, we first mention that for a convergent vector subdivision scheme, one has, similar to (1.2),

$$
S^{n} v(\alpha) \approx\left[f_{1}\left(M^{-n} \alpha\right), \ldots, f_{m}\left(M^{-n} \alpha\right)\right], \quad n \text { large. }
$$

But the limit functions $f_{i}$ are typically highly correlated - and the exact correlation among the component functions $\left(f_{i}\right)$ is implicit to the user of the subdivision scheme. There is a concept called the rank of a subdivision scheme that basically describes this situation in a rigorous way. For a detailed account of this concept, we recommend the interdependence analysis section of the unpublished article [2]. Here we give an intuitive description of two extreme cases:

[C0] Rank $=m$. A rank $m$ scheme is essentially $m$ entirely unrelated convergent scalar subdivision schemes "put together". In this case, the $f_{i}, i=1, \ldots, m$, are essentially $m$ entirely unrelated functions.

[C1] Rank $=1$. The limit functions $f_{i}, i=1, \ldots, m$, of a rank 1 scheme are essentially just one function: each $f_{i}$, redefined based on a suitable renormalization in the subdivision process (see below for one example), must be a linear combination of the derivatives of a single function $f$.

From our point of view, the rank 1 scheme seems like the only kind of vector subdivision scheme of direct interest in this application. Any vector subdivision scheme with a rank $>1$ is essentially the "putting together" of more than one unrelated schemes. Notice then a fine point of the aforementioned comment [C1] pertaining to the rank 1 scheme: the exact linear combination mentioned in [C1] depends on the subdivision scheme itself. For two different rank 1 schemes, the corresponding linear combinations may be entirely different. It is in this sense that we say that the numerical values generated by a general vector subdivision scheme do not possess precise geometric meanings.

Hermite subdivision scheme: vector subdivision scheme with a prescribed geometric meaning. To overcome the above-mentioned difficulty in applications to free-form surfaces, in this article we introduce and study a special class of vector subdivision schemes with a "prescribed geometric meaning" made precise in Definition 1.1 In the case of $s=1, M=[2]$, these subdivision schemes 
behave in such a way that for every $v$, there is a $C^{m-1}$ function $f$ such that, for $l=0, \ldots, m-1$,

$$
\left(S^{n} v\right)(\alpha)_{l+1} \approx f^{(l)}\left(2^{-n} \alpha\right) \times 2^{-n l}, \quad n \text { large. }
$$

Consequently, the components of $\left(S^{n} v\right)(\alpha)$ have rather precise meanings, namely, "approximate position", "approximate gradient", "approximate curvature", etc., of a point on the limit curve.

Such subdivision schemes can be viewed as a generalization of the more wellstudied interpolatory Hermite subdivision schemes [24, 10, 7, 16, 5, 17, in which

$$
\text { ' } \approx \text { ' and ' } n \text { large' }
$$

above are replaced by

$$
'=\text { and ' } \forall n '
$$

respectively. Figure 1 1 shows an interpolatory and a noninterpolatory Hermite scheme in action. Starting from a set of Hermite data defined on integers, a Hermite subdivision scheme successively introduces Hermite data at the half-integers, the quarter-integers and so on. The distinction between an interpolatory and a noninterpolatory scheme should be evident from the figures.

It is well known to geometric modelling practitioners that interpolatory schemes often produce "unfair" surfaces - surfaces with unwanted wiggles or undulations (see Figure 2(c)) - and noninterpolatory schemes are much preferred in geometric modelling applications.

Relating (1.4) to (1.3) in 1-D with $M=[2]$, a Hermite subdivision scheme is one such that

$$
\left[f_{1}, \ldots, f_{m}\right]=[f, 0, \ldots, 0]
$$

and if we modify the definition of $\left[f_{1}, \ldots, f_{m}\right]$ from " $\lim _{n \rightarrow \infty} S^{n} v$ " to

$$
\text { " } \lim _{n \rightarrow \infty} S^{n} v \times \operatorname{diag}\left[1,2^{n}, \ldots, 2^{(m-1) n}\right] ",
$$

then

$$
\text { "the renormalized }\left[f_{1}, \ldots, f_{m}\right] "=\left[f, f^{\prime}, \ldots, f^{(m-1)}\right] \text {. }
$$

(The above will be formally defined in Definition (1.1).)

Other Possibilities. Hermite schemes are not the only schemes that serve our needs. In Definition 3.1, we define another kind of vector subdivision schemes termed Lagrange type schemes, for which the relation corresponding to (1.5) becomes

$$
\left[f_{1}, \ldots, f_{m}\right]=[f, f, \ldots, f] \text {. }
$$

However, as explained in Section 3.1 we believe that there is no fundamental difference between Hermite and Lagrange schemes.

Symmetry. All the schemes for free-form subdivision surfaces published in 6, 1, 23, 9, 29, 22, 21, 26 are based on a scalar (i.e., $m=1$ ) subdivision scheme of the form (1.1) with a certain symmetry property (together with special subdivision rules at extraordinary vertices). Symmetry occurs to be a very fundamental requirement for applications in free-form surfaces, because on a manifold there is not a natural choice of a local coordinate system and hence it seems necessary to apply a subdivision scheme which is insensitive to such a choice.

For a general vector subdivision scheme (1.1), however, it is unclear what symmetry means. 
Interpolatory

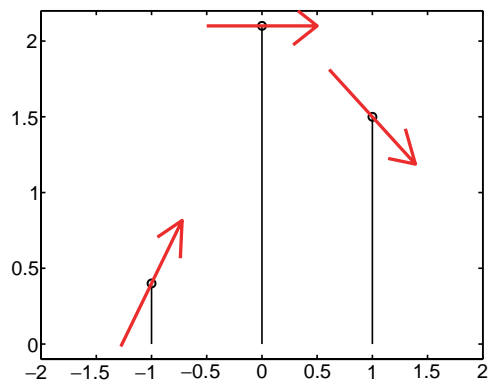

(initial Hermite data)

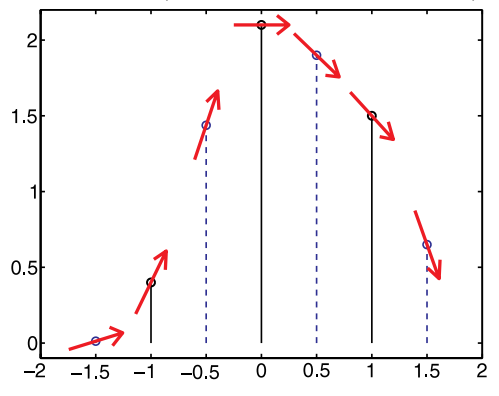

(one step)

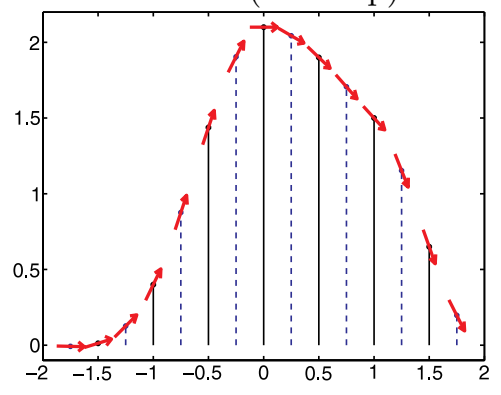

(two steps)

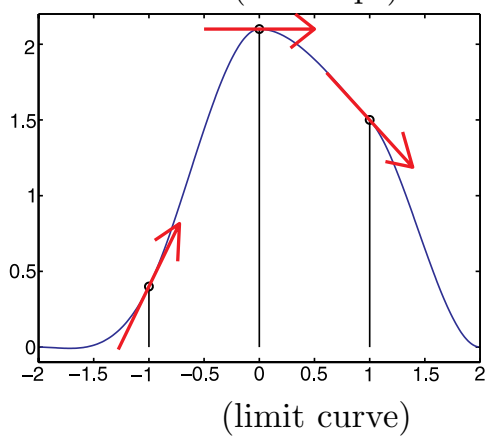

Noninterpolatory

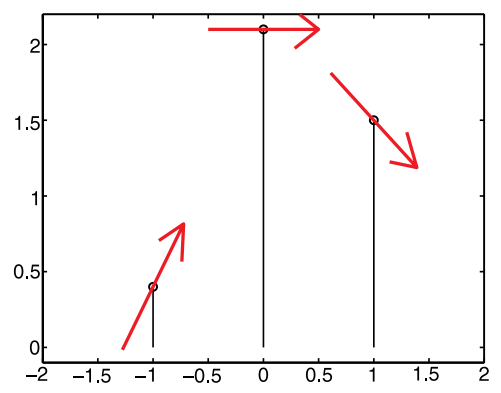

(initial Hermite data)
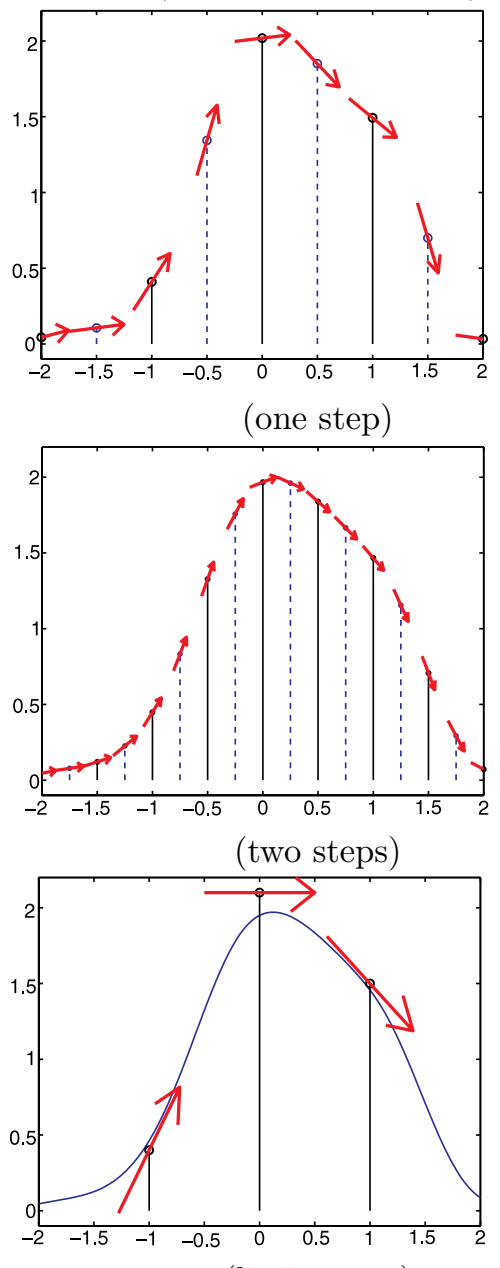

(limit curve)

FiguRE 1. 1-D interpolatory and noninterpolatory Hermite subdivision

This second issue is, after all, highly correlated to the first one: it is unclear what symmetry may mean to a vector subdivision scheme precisely because we do not 


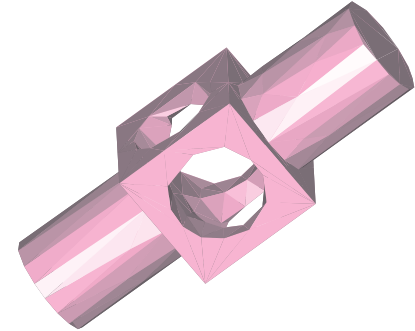

(a) Coarse mesh

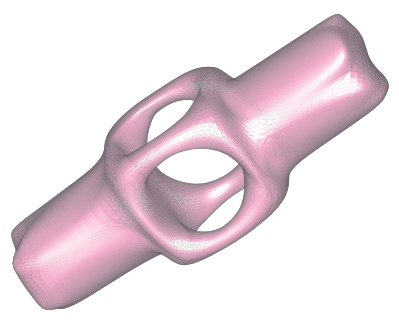

(b) Loop [23] - noninterpolatory

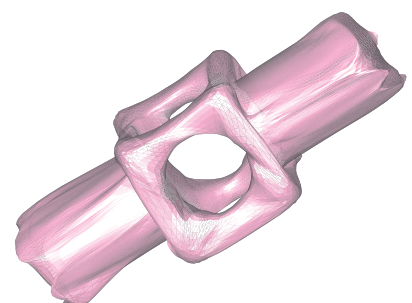

(c) Butterfly 29] -interpolatory

FiguRE 2. A noninterpolatory and an interpolatory (scalar) subdivision scheme applied to a very irregular coarse mesh. It is a well-known experience that interpolatory schemes typically produce surfaces which are less "fair" than those produced by noninterpolatory schemes.

have a "geometric interpretation" to the data $S^{n} v(\alpha)$ produced by the subdivision process. But if a vector subdivision scheme is of the Hermite type considered in the paper, then the meaning of symmetry becomes immediately clear. See Section 2.1 .

Having a precise "geometric meaning" and a natural symmetry property that comes along with it, the vector subdivision schemes constructed in this article are currently being applied to construct free-form subdivision surfaces [28].

1.1. Notations. Let $\Lambda_{r}:=\left\{\mu \in \mathbb{N}_{0}^{s}:|\mu| \leq r\right\}$ and by $\# \Lambda_{r}$ we denote the cardinality of the set $\Lambda_{r}$. Now the elements in $\Lambda_{r}$ can be ordered according to the lexicographic order. That is, $\left(\nu_{1}, \ldots, \nu_{s}\right)$ is less than $\left(\mu_{1}, \ldots, \mu_{s}\right)$ in lexicographic order if $|\nu|<|\mu|$ or $\nu_{j}=\mu_{j}$ for $j=1, \ldots, i-1$ and $\nu_{i}<\mu_{i}$ for some $i$. The set $\Lambda_{r}$ is always ordered in the lexicographic order in this paper with the default first element being 0 . When we write $u=\left(u_{\mu}\right)_{\mu \in \Lambda}$, it means $u$ is a (row or column) vector with its entries ordered lexicographically. A similar comment applies to $\# \Lambda_{r} \times \# \Lambda_{r}$ matrices.

For an $s \times s$ matrix $E$, define the $\left(\# \Lambda_{r}\right) \times\left(\# \Lambda_{r}\right)$ matrix $\mathcal{S}\left(E, \Lambda_{r}\right)([12])$ by the unique matrix that satisfies

$$
g=f(E \cdot) \text { implies } \partial^{\leq r} g=\partial^{\leq r} f(E \cdot) \mathcal{S}\left(E, \Lambda_{r}\right)
$$

where $\partial^{\leq r} f(x)$ is the row vector of length $\# \Lambda_{r}$ with entries $D^{\mu} f(x), \mu \in \Lambda_{r}$. In other words, $\mathcal{S}\left(E, \Lambda_{r}\right)$ measures how Hermite data change under a linear change of variables. From this interpretation, we get $\mathcal{S}\left(E_{1} E_{2}, \Lambda_{r}\right)=\mathcal{S}\left(E_{1}, \Lambda_{r}\right) \mathcal{S}\left(E_{2}, \Lambda_{r}\right)$ and $\mathcal{S}\left(E^{-1}, \Lambda_{r}\right)=\left[\mathcal{S}\left(E, \Lambda_{r}\right)\right]^{-1}$, provided that $E$ is invertible.

For a vector space $B$, denote by $[B]^{m \times n}$ the vector space of all matrices of elements in $B$, equipped with the cartesian vector space structure. Define $[B]^{m}:=$ $[B]^{m \times 1}$. If $B$ is a normed space with norm $\|\cdot\|_{B}$, then $[B]^{m \times n}$ is a normed space with norm $\|v\|_{[B]^{m \times n}}:=\left\|\left(\left\|v_{i, j}\right\|_{B}\right)_{1 \leq i \leq m, 1 \leq j \leq n}\right\|$, where the outer $\|\cdot\|$ is any norm in $\mathbb{R}^{m \times n}$. If $\left(B,\|\cdot\|_{B}\right)$ is a Banach space, then so is $\left([B]^{m \times n},\|\cdot\|_{[B]^{m \times n}}\right)$.

We denote by $l\left(\mathbb{Z}^{s}\right)$ and $L\left(\mathbb{R}^{s}\right)$ the vector spaces of all complex valued sequences and functions defined on $\mathbb{Z}^{s}$ and $\mathbb{R}^{s}$. The space of finitely supported sequences is denoted by $l^{0}\left(\mathbb{Z}^{s}\right)$. 
Throughout this paper, $M$ is an isotropic dilation matrix. If $f: \mathbb{R}^{s} \rightarrow \mathbb{R}$ is a smooth function, the notation $\left.\left[\partial^{\leq r} f\right]\right|_{M^{-n} \mathbb{Z}^{s}}$ refers to the element in $\left[l\left(\mathbb{Z}^{s}\right)\right]^{1 \times \# \Lambda_{r}}$ defined by $\left(\left.\left[\partial^{\leq r} f\right]\right|_{M^{-n} \mathbb{Z}^{s}}\right)(\alpha)=\left[\partial^{\leq r} f\right]\left(M^{-n} \alpha\right)$.

1.2. Hermite type subdivision schemes. We now formally define the object of interest in this article.

Definition 1.1. A subdivision scheme $S:=S_{\mathbf{a}, M}$ is of Hermite type of order $r$ if (i) $m=\# \Lambda_{r}$ for some $r \geq 0$, and for any initial sequence $v \in\left[l^{0}\left(\mathbb{Z}^{s}\right)\right]^{1 \times m}$ there exists $f_{v} \in C^{r}\left(\mathbb{R}^{s}\right)$ such that

$$
\lim _{n \rightarrow \infty}\left\|\left.\left[\partial^{\leq r} f_{v}\right]\right|_{M^{-n} \mathbb{Z}^{s}}-v_{n} \mathcal{S}\left(M^{n}, \Lambda_{r}\right)\right\|_{\left[l^{\infty}\left(\mathbb{Z}^{s}\right)\right]^{1 \times m}}=0
$$

where $v_{n}=S^{n} v$, (ii) $f_{v} \neq 0$ for some $v \neq 0$.

Having formally defined Hermite type subdivision schemes, the mission of the rest of the article is as follows.

Organization. In Section 2, we derive a constructive condition for Hermite type subdivision masks. The section encompasses the key mathematical contribution of this article; namely, we establish Theorem 2.2. This result utilizes a major technical result in the so-called strong convergence theory of vector refinement equations 13 . Theorem 4.3] to derive an implementable algebraic condition on the subdivision mask of a Hermite type subdivision scheme. Section 2.1 discusses condition for symmetry, which is crucial for applying Hermite type subdivision schemes in the free-form surface setting 28. In Section 3 we set up a computational framework for constructing Hermite type subdivision masks, and we report some computational findings. While this computational framework is based on the analytical results in Section 2, we sketch a different approach for constructing Hermite type subdivision schemes in 1-D based on multiple-knot $B$-splines. In Section 4 we give several concluding remarks.

\section{AnAlysis}

We begin with a road map of this theory section. Since the subdivision mask determines everything about a subdivision scheme, the ultimate question for us is: what are the necessary and/or sufficient condition(s) satisfied by the mask of a Hermite type subdivision scheme?

This section addresses the above question in the following steps:

- Old components. Each of the following implications is known in the literature and is not specific to a Hermite type subdivision scheme.

\begin{tabular}{|c|c|}
\hline$\Longrightarrow$ & $\begin{array}{l}S_{\mathbf{a}, M} \text { is a } W_{p}^{k} \text { smooth vector subdivision scheme with multiplicity } m \\
\text { impulse response of } S_{\mathbf{a}, M}, \phi \text {, is refinable with mask a and } \\
\phi \in\left[W_{p}^{k}\left(\mathbb{R}^{s}\right)\right]^{m \times 1}\end{array}$ \\
\hline$\Longrightarrow$ & $\operatorname{span}\left\{\phi_{i}(\cdot-\alpha): \alpha \in \mathbb{Z}^{s}, i=1, \ldots, m\right\} \supseteq \Pi_{k} \quad$ (accuracy order) \\
\hline$\Longleftrightarrow$ & $\operatorname{span}\left\{\psi(\cdot-\alpha): \alpha \in \mathbb{Z}^{s}\right\} \supseteq \Pi_{k}$ \\
\hline & $\hat{\psi}=\hat{y} \hat{\phi}$, for some $y \in\left[l^{0}\left(\mathbb{Z}^{s}\right)\right]^{1 \times m}$ (super-function) \\
\hline$\Longleftrightarrow$ & $\psi$ satisfies $D^{\mu} \hat{\psi}(2 \pi \beta)=0, \beta \neq 0,|\mu| \leq r \quad$ (Strang-Fix Condition) \\
\hline$\phi$ has stable shifts & $\left\{\begin{array}{l}D^{\mu}\left[\hat{y}\left(M^{T} \cdot\right) \hat{a}(\cdot)\right](0)=D^{\mu} \hat{y}(0) \quad \forall|\mu| \leq r, \\
D^{\mu}\left[\hat{u}\left(M^{T}\right) \hat{a}(\cdot)\right](2 \pi \beta)\end{array}\right.$ \\
\hline & $\begin{array}{l}\left(D^{\mu}\left[y\left(M M^{2} \cdot\right) a(\cdot)\right](2 \pi \beta)=0\right. \\
\text { (sum rule conditions w.r.t. } y \text { ) }\end{array}$ \\
\hline
\end{tabular}


- New components. We use a result in the strong convergence theory of vector refinement equations [13, Theorem 4.3] to show that if a mask a satisfies the sum rule conditions w.r.t. to a $y$ with a specific structure (see (2.9)), together with a standard spectral condition on the mask (see (2.8)), then $S_{a, M}$ is an order $r$ Hermite type subdivision scheme.

We now pursue these components in detail.

It is clear that the limit function $f_{v}$ in Definition 1.1, when it exists, is unique. In fact,

$$
f_{v}=\sum_{\alpha} v(\alpha) \phi(\cdot-\alpha)
$$

where $\phi=\left(\phi_{\mu}\right)_{\mu \in \Lambda_{r}}$ (as a column vector) is the "impulse response" of the subdivision scheme: $\phi_{\mu}:=f_{\delta e_{\mu}}$; here $e_{\mu}$ is the $\mu$-th coordinate unit vector in $\mathbb{R}^{1 \times \# \Lambda_{r}}$. The vector $\phi$ satisfies the well-studied two-scale refinement equation

$$
\phi(x)=\sum_{\alpha \in \mathbb{Z}^{s}} \mathbf{a}(\alpha) \phi(M x-\alpha) .
$$

It is well known from the theory of refinement equations that if equation (2.1) has a smooth solution $\phi \in\left[C^{r}\left(\mathbb{R}^{s}\right)\right]^{m}$, then

$$
\operatorname{span}\left\{\phi(\cdot-\alpha): \alpha \in \mathbb{Z}^{s}\right\} \supseteq \Pi_{r}
$$

and if in addition $\operatorname{span}\left\{\hat{\phi}\left(2 \pi\left(M^{T}\right)^{-1} \alpha+2 \pi \beta\right): \beta \in \mathbb{Z}^{s}\right\}=\mathbb{C}^{m}$ for all $\alpha \in \mathbb{Z}^{s}$, then the mask a must satisfy the sum rules of order $r+1$ (see [10. Theorem 2.4] and [13]): $\exists y \in\left[l^{0}\left(\mathbb{Z}^{s}\right)\right]^{1 \times m}$ such that $\hat{y}(0) \neq 0$,

$$
D^{\mu}\left[\hat{y}\left(M^{T} \cdot\right) \hat{\mathbf{a}}(\cdot)\right](0)=D^{\mu} \hat{y}(0) \quad \forall|\mu| \leq r
$$

and

$$
D^{\mu}\left[\hat{y}\left(M^{T} \cdot\right) \hat{\mathbf{a}}(\cdot)\right](2 \pi \beta)=0 \quad \forall|\mu| \leq r, \beta \in\left(M^{T}\right)^{-1} \mathbb{Z}^{s} \backslash \mathbb{Z}^{s} .
$$

Conversely, if a satisfies the sum rules of order $r+1$, then (2.2) must be true ([10. Theorem 2.4]). As we will see, the Hermite property of a subdivision mask a is related to the structure of the vector $y$ above; from this the vector $y$ is partially known and the mask a can be determined from (2.3)-(2.4) up to certain degrees of freedom.

Given a mask $\mathbf{a} \in\left[l^{0}\left(\mathbb{Z}^{s}\right)\right]^{m \times m}$, recall that the associated cascade operator $Q:=$ $Q_{\mathbf{a}, M}$ is defined by

$$
Q f=\sum_{\alpha \in \mathbb{Z}^{s}} \mathbf{a}(\alpha) f(M \cdot-\alpha)
$$

Let $f$ be a length $m$ column vector of tempered distributions. We say that $f$ satisfies the moment conditions of order $r+1$ with respect to $y \in\left[l^{0}\left(\mathbb{Z}^{s}\right)\right]^{1 \times m}$ (see [13]) if

$$
\hat{y}(0) \hat{f}(0)=1 \quad \text { and } \quad D^{\mu}[\hat{y}(\cdot) \hat{f}(\cdot)](2 \pi \beta)=0, \quad \mu \in \Lambda_{r}, \quad \beta \in \mathbb{Z}^{s} \backslash\{0\} .
$$

Assume that (2.3) is satisfied with some vector $y$. We say that the cascade algorithm associated with mask a and dilation matrix $M$ converges in $C^{r}\left(\mathbb{R}^{s}\right)$ if for any compactly supported function vector $f \in\left[C^{r}\left(\mathbb{R}^{s}\right)\right]^{m}$ satisfying the moment conditions of order $r+1$ with respect to $y$, the sequence $\left(Q_{\mathbf{a}, M}^{n} f\right)_{n=0}^{\infty}$ is a Cauchy sequence in $\left[C^{r}\left(\mathbb{R}^{s}\right)\right]^{m}$; consequently, there exists $\phi \in\left[C^{r}\left(\mathbb{R}^{s}\right)\right]^{m}$ such that

$$
\lim _{n \rightarrow \infty}\left\|Q_{\mathbf{a}, M}^{n} f-\phi\right\|_{\left[C^{r}\left(\mathbb{R}^{s}\right)\right]^{m}}=0 .
$$


In this case the limit $\phi$ is a solution of the refinement equation (2.1) and moreover, (2.3) -(2.4) are satisfied (see [13]).

It is well known that $(2.2)$ is equivalent to the existence of a $y \in\left[l^{0}\left(\mathbb{Z}^{s}\right)\right]^{1 \times m}$ such that (2.6) holds with $f$ being replaced by $\phi$. This vector $y$, in vague terms, determines how the function vector $\phi$ reproduces $\Pi_{r}$ : in fact (2.6) is equivalent to saying that the so-called super-function $g:=y * f:=\sum_{\alpha} y(\alpha) f(\cdot-\alpha)$ satisfies $\sum_{\alpha} p(\alpha) g(\cdot-\alpha) \in \Pi_{r}$ for all $p \in \Pi_{r}$; alternatively,

$$
\begin{aligned}
p *(y * f) & =(p * y) * f \\
& =\sum_{\beta \in \mathbb{Z}^{s}}[p(\beta-i D) \hat{y}](0) f(\cdot-\beta) \in \Pi_{r} \quad \forall p \in \Pi_{r} .
\end{aligned}
$$

(Here $D=\left[D_{1}, \ldots, D_{s}\right]$ is a vector of differential operators, and for a polynomial $p \in \Pi_{r}, p(\beta-i D)$ denotes the differential operator $\sum_{\nu \geq 0}\left(D^{\nu} p\right)(\beta) / \nu !(-i D)^{\nu}$.) In addition to (2.6) , if $D^{\mu}[\hat{y}(\cdot) \hat{f}(\cdot)](0)=0$ for all $\mu \in \Lambda_{r} \backslash\{0\}$, then $p=p *(y * f)$ for all $p \in \Pi_{r}$.

Being an iterative algorithm, a spectral quantity $\nu_{\infty}(\mathbf{a}, M)$ determines whether the cascade algorithm with mask a and dilation matrix $M$ converges in $C^{k}$. For a definition of $\nu_{p}(\mathbf{a}, M), p \in[1, \infty]$, see [13. Section 4]. The quantity $\nu_{p}(\mathbf{a}, M)$ plays a fundamental role in the study of the convergence of vector cascade algorithms and the smoothness of refinable function vectors ([13). For example, the cascade algorithm associated with mask a and dilation matrix $M$ converges in the Sobolev space $W_{p}^{k}\left(\mathbb{R}^{s}\right)(1 \leq p \leq \infty)$ if and only if $\nu_{p}(\mathbf{a}, M)>k$. Let $\phi$ be the refinable function vector with the mask $\mathbf{a}$ and the dilation matrix $M$. Then one always has $\nu_{p}(\phi) \geq \nu_{p}(\mathbf{a}, M)$. If in addition the shifts of $\phi$ are stable, then one has $\nu_{p}(\phi)=\nu_{p}(\mathbf{a}, M)$. For detailed discussion on these issues, see 13. When a cascade algorithm converges in $C^{r}\left(\mathbb{R}^{s}\right)$, the same vector $y$ above essentially determines which are the initial function vectors $f$ for which the cascade sequence $\left(Q^{n} f\right)_{n=1}^{\infty}$ converges:

Theorem 2.1 ([13 Theorem 4.3]). Let $\mathbf{a} \in\left[l^{0}\left(\mathbb{Z}^{s}\right)\right]^{m \times m}$, let $M$ be an $s \times s$ isotropic dilation matrix, let $y \in\left[l^{0}\left(\mathbb{Z}^{s}\right)\right]^{1 \times m}$ be such that $\hat{y}(0) \neq 0$ and let (2.3) hold for a nonnegative integer $k$. Then the following are equivalent:

(i) For every compactly supported function vector $f \in\left[C^{k}\left(\mathbb{R}^{s}\right)\right]^{m \times 1}$ such that $f$ satisfies the moment conditions of order $k+1$ with respect to $y,\left(Q_{\mathbf{a}, M}^{n} f\right)_{n=1}^{\infty}$ is a Cauchy sequence in $\left[C^{k}\left(\mathbb{R}^{s}\right)\right]^{m \times 1}$;

(ii) $\nu_{\infty}(\mathbf{a}, M)>k$.

This theorem happens to give a set of simple sufficient conditions for constructing Hermite type subdivision masks. We now present the main result of this section:

Theorem 2.2. Let $M$ be an isotropic dilation matrix and let $\mathbf{a}$ be a mask with multiplicity $m=\# \Lambda_{r}$. Suppose that

$$
\nu_{\infty}(\mathbf{a}, M)>r .
$$

Then $S_{\mathbf{a}, M}$ is a subdivision scheme of Hermite type of order $r$ if a satisfies the sum rules of order $r+1$ with a sequence $y \in\left[l^{0}\left(\mathbb{Z}^{s}\right)\right]^{1 \times \# \Lambda_{r}}$ such that

$$
\frac{(-i D)^{\mu}}{\mu !} \hat{y}(0)=e_{\mu}^{T}, \quad \mu \in \Lambda_{r} .
$$


Proof. Let $\psi$ be a Hermite interpolant of order $r$ with accuracy order $r+1$ (see, e.g., [16, Lemma 2.6]). We observe that $\psi$ satisfies the moment conditions of order $r+1$ with respect to a $y$ which satisfies (2.9). This can be easily seen from (2.7) and the Hermite interpolation property of $\psi$ : for $p(x)=x^{\mu}, \mu \in \Lambda_{r}, p=$ $\sum_{\alpha}[p(\alpha-i D) \hat{y}](0) \psi(\cdot-\alpha)$, but since $D^{\mu} \psi_{\nu}(\alpha)=\delta_{\alpha, 0} \delta_{\mu, \nu}$, we have $\left[(-i D)^{\mu} \hat{y}\right](0)=$ $\left[\partial^{\leq}(\cdot)^{\mu}\right](0)$, which is equivalent to (2.9)). Then since $\nu_{\infty}(\mathbf{a}, M)>r$, by Theorem 2.1 we have $\lim _{n \rightarrow \infty}\left\|Q_{\mathbf{a}, M}^{n} \psi-\phi\right\|_{\left[C^{r}\left(\mathbb{R}^{s}\right)\right]^{m \times 1}}=0$ for some $\phi \in\left[C^{r}\left(\mathbb{R}^{s}\right)\right]^{m \times 1}$.

Recall a basic connection between the cascade algorithm and the subdivision scheme: if $a_{n}=S^{n}\left(\delta I_{m \times n}\right)$, then $Q^{n} \psi=\sum_{\alpha} a_{n}(\alpha) \psi\left(M^{n} \cdot-\alpha\right)$. Let $v \in\left[l^{0}\left(\mathbb{Z}^{s}\right)\right]^{1 \times m}$, $v_{n}:=S^{n} v$, then $v_{n}=\sum_{\beta} v(\beta) a_{n}\left(\cdot-M^{n} \beta\right)$. Then

$$
\begin{aligned}
f_{n}:=\sum_{\alpha \in \mathbb{Z}^{s}} v_{n}(\alpha) \psi\left(M^{n} \cdot-\alpha\right) & =\sum_{\alpha \in \mathbb{Z}^{s}} \sum_{\beta \in \mathbb{Z}^{s}} v(\beta) a_{n}\left(\alpha-M^{n} \beta\right) \psi\left(M^{n} \cdot-\alpha\right) \\
& =\sum_{\beta \in \mathbb{Z}^{s}} v(\beta)\left(Q^{n} \psi\right)(\cdot-\beta) .
\end{aligned}
$$

Therefore, if $f:=\sum_{\alpha} v(\alpha) \phi(\cdot-\alpha)$, then

$$
\lim _{n \rightarrow \infty}\left\|f_{n}-f\right\|_{C^{r}\left(\mathbb{R}^{s}\right)}=0
$$

and

$$
\lim _{n \rightarrow \infty}\left\|D^{\mu} f_{n}-D^{\mu} f\right\|_{L^{\infty}}=0, \forall \mu \in \Lambda_{r} .
$$

If we denote by $\partial^{\leq r} \psi(x)$ the $\# \Lambda_{r} \times \# \Lambda_{r}$ matrix with the $\mu$-th row equals to $\partial^{\leq r} \psi_{\mu}(x)$, then since $\psi$ is a Hermite interpolant, $\partial^{\leq r} \psi(\alpha)=I_{\# \Lambda_{r} \times \# \Lambda_{r}} \delta(\alpha), \forall \alpha \in$ $\mathbb{Z}^{s}$. By (1.6), we have

$$
\partial^{\leq r} f_{n}\left(M^{-n} \alpha\right)=\sum_{\beta \in \mathbb{Z}^{s}} v_{n}(\beta)\left(\partial^{\leq r} \psi\right)(\alpha-\beta) \mathcal{S}\left(M^{n}, \Lambda_{r}\right)=v_{n}(\alpha) \mathcal{S}\left(M^{n}, \Lambda_{r}\right) .
$$

But

$$
\begin{array}{rl}
\| \partial^{\leq r} & f\left(M^{-n} \cdot\right)-v_{n}(\cdot) \mathcal{S}\left(M^{n}, \Lambda_{r}\right) \|_{\left[l^{\infty}\left(\mathbb{Z}^{s}\right)\right]^{1 \times m}} \\
& =\left\|\partial^{\leq r} f\left(M^{-n} \cdot\right)-\partial^{\leq r} f_{n}\left(M^{-n} \cdot\right)\right\|_{\left[L^{\infty}\left(\mathbb{R}^{s}\right)\right]^{1 \times m}} \\
& \leq \max _{\mu \in \Lambda_{r}}\left\|D^{\mu} f_{n}-D^{\mu} f\right\|_{L^{\infty}} \rightarrow 0, \text { as } n \rightarrow \infty .
\end{array}
$$

So a satisfies condition (i) of Definition 1.1.

The condition $\nu_{\infty}(\mathbf{a}, M)>r$ implies, by Theorem 2.1 that $\operatorname{span}\{\phi(\cdot-\beta): \beta \in$ $\left.\mathbb{Z}^{s}\right\} \supseteq \Pi_{r}$, which implies $\phi \neq 0$. Thus condition (ii) of Definition 1.1] is also satisfied by $\mathbf{a}$.

Note that the assumption $\nu_{\infty}(\mathbf{a}, M)>r$ implies that a must satisfy the sum rules of order $r+1$ with some sequence $y$ and, in fact, the values $(-i D)^{\mu} \hat{y}(0), \mu \in \Lambda_{r}$, of such a sequence $y$ are uniquely determined by a and $M$ up to a scalar multiplicative constant. See 13 for more details on the analysis of vector cascade algorithms. The condition in (2.9) on the sequence $y$ was first introduced in [10] for the purpose of studying refinable Hermite interpolants.

2.1. Symmetry. Let $G$ be a finite subset of integer matrices whose determinants are \pm 1 . This would in particular imply that each element in $G$ induces a linear isomorphism on $\mathbb{Z}^{s}$. We say that $G$ is a symmetry group with respect to a dilation matrix $M([12,11])$ if $G$ forms a group under matrix multiplication and

$$
M E M^{-1} \in G \text { for all } E \in G \text {. }
$$


This definition is essential for the following

Proposition 2.3. Let $S_{\mathbf{a}, M}$ be a Hermite type subdivision scheme of order $r$, and let $\phi=\left(\phi_{\mu}\right)_{\mu \in \Lambda_{r}}$ be defined by $\phi_{\mu}=S_{\mathbf{a}, M}^{\infty}\left(\delta e_{\mu}\right)$. Let $G$ be a symmetry group with respect to the dilation matrix $M$. The following are equivalent:

(1) The operator $\mathcal{I}_{\phi}:\left[l\left(\mathbb{Z}^{s}\right)\right]^{1 \times \# \Lambda_{r}} \rightarrow C^{r}\left(\mathbb{R}^{s}\right)$ defined by $\mathcal{I}_{\phi}:=\sum_{\alpha} v(\alpha) \phi(\cdot-\alpha)$ respects change of variables by $E \in G$ in the following sense: For any $F \in$ $C^{r}$ and $G:=F(E \cdot), E \in G, \tilde{F}:=\mathcal{I}_{\phi}\left(\left.\partial^{\leq r} F\right|_{\mathbb{Z}^{s}}\right)$ and $\tilde{G}:=\mathcal{I}_{\phi}\left(\left.\partial^{\leq r} G\right|_{\mathbb{Z}^{s}}\right)$ are again related by $\tilde{G}:=\tilde{F}(E \cdot)$.

(2) $\phi(E x)=\mathcal{S}\left(E, \Lambda_{r}\right) \phi(x) \forall E \in G, x \in \mathbb{R}^{s}$.

If, in addition, we assume that 1 is a simple and dominant eigenvalue of the matrix

$$
J_{0}:=\sum_{\beta \in \mathbb{Z}^{s}} \mathbf{a}(\beta) /|\operatorname{det} M|
$$

and the first entry of its nonzero eigenvector for the eigenvalue 1 is nonzero, then both (1) and (2) are implied by the following condition on the mask $\mathbf{a}$ :

(3) $\mathbf{a}(E \alpha)=\mathcal{S}\left(M^{-1} E M, \Lambda_{r}\right) \mathbf{a}(\alpha) \mathcal{S}\left(E^{-1}, \Lambda_{r}\right) \quad \forall \alpha \in \mathbb{Z}^{s}, E \in G$.

Proof. Assuming (2), $\tilde{G}=\sum_{\alpha} \partial^{\leq r} G(\alpha) \phi(\cdot-\alpha)=\sum_{\alpha} \partial^{\leq r} F(E \alpha) \mathcal{S}\left(E, \Lambda_{r}\right) \phi(\cdot-\alpha)$ $=\sum_{\alpha} \partial^{\leq r} F(E \alpha) \phi(E \cdot-E \alpha)=\sum_{\alpha} \partial^{\leq r} F(\alpha) \phi(E \cdot-\alpha)=\tilde{F}(E \cdot)$. Thus (2) $\Rightarrow(1)$. Conversely, pick any Hermite interpolant $F=\left(F_{\nu}\right)_{\nu \in \Lambda_{r}}$. Then $\tilde{F}_{\nu}=$ $\mathcal{I}_{\phi}\left(\left.\partial^{\leq r} F_{\nu}\right|_{\mathbb{Z}^{s}}\right)=\phi_{\nu}$ and $\tilde{G}_{\nu}=\mathcal{I}_{\phi}\left(\left.\partial^{\leq r} F_{\nu}(E \cdot)\right|_{\mathbb{Z}^{s}}\right)=\partial^{\leq r} F_{\nu}(0) \mathcal{S}\left(E, \Lambda_{r}\right) \phi$, so $\phi_{\nu}(E \cdot)$ $=\tilde{F}_{\nu}(E \cdot)=\tilde{G}_{\nu}=e_{\nu} \mathcal{S}\left(E, \Lambda_{r}\right) \phi$, for all $\nu \in \Lambda_{r}$, which is equivalent to (2).

$(3) \Rightarrow(2)$ Let $\phi_{E}(x):=\mathcal{S}\left(E^{-1}, \Lambda_{r}\right) \phi(E x)$. We claim that

$$
\phi_{E}=Q_{\mathbf{a}, M} \phi_{M E M^{-1}} \quad \forall E \in G \text {. }
$$

This relies on the basic assumption (2.10), which implies that for any $E \in G$, $M E M^{-1}$ is an isomorphism on $\mathbb{Z}^{s}$, so

$$
\begin{aligned}
Q_{\mathbf{a}, M} & \phi_{M E M^{-1}} \\
& =\sum_{\alpha} \mathbf{a}(\alpha) \phi_{M E M^{-1}}(M \cdot-\alpha) \\
& =\sum_{\alpha} \mathbf{a}(\alpha) \mathcal{S}\left(M E^{-1} M^{-1}, \Lambda_{r}\right) \phi\left(M E \cdot-M E M^{-1} \alpha\right) \\
& =\sum_{\beta} \mathbf{a}\left(M E^{-1} M^{-1} \beta\right) \mathcal{S}\left(M E^{-1} M^{-1}, \Lambda_{r}\right) \phi\left(M E \cdot-M E M^{-1} M E^{-1} M^{-1} \beta\right) \\
& =\sum_{\alpha} \mathbf{a}\left(M E^{-1} M^{-1} \alpha\right) \mathcal{S}\left(M E^{-1} M^{-1}, \Lambda_{r}\right) \phi(M E \cdot-\alpha) .
\end{aligned}
$$

Let $E^{\prime}:=M E^{-1} M^{-1} \in G$. By (3), we have

$$
\mathbf{a}\left(E^{\prime} \alpha\right) \mathcal{S}\left(E^{\prime}, \Lambda_{r}\right)=\mathcal{S}\left(M^{-1} E^{\prime} M, \Lambda_{r}\right) \mathbf{a}(\alpha)
$$

or

$$
\mathbf{a}\left(M E^{-1} M^{-1} \alpha\right) \mathcal{S}\left(M E^{-1} M^{-1}, \Lambda_{r}\right)=\mathcal{S}\left(E^{-1}, \Lambda_{r}\right) \mathbf{a}(\alpha) .
$$

So (2.12) equals $\sum_{\alpha} \mathcal{S}\left(E^{-1}, \Lambda_{r}\right) \mathbf{a}(\alpha) \phi(M E \cdot-\alpha)=\phi_{E}$ and the claim is proved.

Iterating (2.11) we have $\phi_{E}=Q_{\mathbf{a}, M}^{n} \phi_{M^{n} E M^{-n}}$ for all $E \in G$ and $n$. Since $G$ is a finite group and $M^{-n} E M^{n} \in G$ for all $n \in \mathbb{N}$, there must exist a positive integer $\ell$ such that $M^{-\ell} E M^{\ell}=E$. Consequently, we have $Q_{a, M}^{\ell} \phi_{E}=\phi_{M^{-\ell} E M^{\ell}}=\phi_{E}$ 
for some positive integer $\ell$. Since 1 is a simple dominant eigenvalue of the matrix $J_{0}$, the same can be said of $J_{0}^{n}$ for all $n \in \mathbb{N}$. If $J_{0}^{n}$ has 1 as a simple dominant eigenvalue, then $\phi$ is the unique solution, up to a scalar multiplicative constant, to the refinement equation $Q_{a, M}^{n} \phi=\phi$.

On the other hand, it follows from the refinement equation that $\hat{\phi}(0)$ and $\widehat{\phi_{E}}(0)$ are eigenvectors of the matrices $J_{0}$ and $J_{0}^{\ell}$, respectively. Since 1 is a simple eigenvalue of $J_{0}^{\ell}$, we must have $\widehat{\phi_{E}}(0)=c \hat{\phi}(0)$ for some complex number $c \in \mathbb{C}$ since $J_{0}^{\ell} \widehat{\phi_{E}}(0)=\widehat{\phi_{E}}(0)$ and $J_{0}^{n} \hat{\phi}(0)=\hat{\phi}(0)$. By the definition of $\phi_{E}$, we have $\widehat{\phi_{E}}(0)=S\left(E^{-1}, \Lambda_{r}\right) \hat{\phi}(0)$ by $|\operatorname{det} E|=1$. By our assumption, the first entry in the vector $\hat{\phi}(0)$ is nonzero; that is, $e_{1}^{T} \hat{\phi}(0) \neq 0$. Note that the first row of the matrix $S\left(E^{-1}, \Lambda_{r}\right)$ is $e_{1}^{T}$. We see that $e_{1}^{T} \widehat{\phi_{E}}(0)=e_{1}^{T} \hat{\phi}(0) \neq 0$. Therefore, it follows from $\widehat{\phi_{E}}(0)=c \hat{\phi}(0)$ that $c$ must be 1 . Hence, we conclude that we must have $\phi_{E}=\phi$ by $Q_{a, M}^{\ell} \phi_{E}=\phi_{E}$ and $\widehat{\phi_{E}}(0)=\hat{\phi}(0)$. In other words, (2) holds.

Discussion. It is not known to us whether (2) implies (3), although we conjecture that it is the case. Indeed, we have

$$
\begin{aligned}
\phi(x) & =\sum_{\alpha} \mathbf{a}(\alpha) \phi(M x-\alpha) \\
& =\sum_{\alpha} \mathbf{a}\left(M E M^{-1} \alpha\right) \phi\left(M x-M E M^{-1} \alpha\right) \quad(\text { by [2.10) }) \\
& =\sum_{\alpha} \mathbf{a}\left(M E M^{-1} \alpha\right) \phi\left(M E M^{-1}\left(M E^{-1} x-\alpha\right)\right) \\
& =\sum_{\alpha} \mathbf{a}\left(M E M^{-1} \alpha\right) \mathcal{S}\left(M E M^{-1}, \Lambda_{r}\right) \phi\left(M E^{-1} x-\alpha\right) \quad(\text { by (2.10) and (2)) } .
\end{aligned}
$$

Let $x^{\prime}=E^{-1} x$. We have $\phi\left(E x^{\prime}\right)=\sum_{\beta} \mathbf{a}\left(M E M^{-1} \beta\right) \mathcal{S}\left(M E M^{-1}, \Lambda_{r}\right) \phi\left(M x^{\prime}-\beta\right)$. Then, by (2),

$$
\phi(x)=\sum_{\beta} \mathcal{S}\left(E^{-1}, \Lambda_{r}\right) \mathbf{a}\left(M E M^{-1} \beta\right) \mathcal{S}\left(M E M^{-1}, \Lambda_{r}\right) \phi(M x-\beta) .
$$

Now since $\phi$ also satisfies the refinement equation with mask a, if we have the guarantee that $\phi$ satisfies a unique refinement equation, then we have $\mathbf{a}(\beta)=$ $\mathcal{S}\left(E^{-1}, \Lambda_{r}\right) \mathbf{a}\left(M E M^{-1} \beta\right) \mathcal{S}\left(M E M^{-1}, \Lambda_{r}\right)$ for all $\beta \in \mathbb{Z}^{s}$ and $E \in G$. This is equivalent to (3).

We discuss this missing uniqueness condition in Section 4 Notice that if $\phi$ is a Hermite interpolant, then this condition is satisfied; compare [16. Proposition 2.8].

\section{Computational Results}

Based on Theorem 2.2 and Proposition 2.3 and the computational optimization idea in [14], we now propose a computational framework for constructing symmetric Hermite type subdivision schemes.

For a given dimension $s \geq 1$, order $r \geq 0$, dilation matrix $M$ and a symmetry group $G$ w.r.t. $M$ :

(i) Pick a finite $G$-symmetric support of the mask a; i.e., choose $\operatorname{supp}(\mathbf{a}) \subset \mathbb{Z}^{s}$ such that $\alpha \in \operatorname{supp}(\mathbf{a})$ implies $E \alpha \in \operatorname{supp}(\mathbf{a})$, for all $E \in G$.

(ii) Pick a target sum rule of order $k+1 \geq r+1$. 
(iii) Solve the following system of (nonlinear) equations:

$$
\begin{gathered}
\mathbf{a}(E \alpha)=\mathcal{S}\left(M^{-1} E M, \Lambda_{r}\right) \mathbf{a}(\alpha) \mathcal{S}\left(E^{-1}, \Lambda_{r}\right), \quad \alpha \in \mathbb{Z}^{s}, E \in G, \\
D^{\mu}\left[\hat{y}\left(M^{T} \cdot\right) \hat{\mathbf{a}}(\cdot)\right](0)=D^{\mu} \hat{y}(0), \quad|\mu| \leq k, \\
D^{\mu}\left[\hat{y}\left(M^{T} \cdot\right) \hat{\mathbf{a}}(\cdot)\right](2 \pi \beta)=0, \quad|\mu| \leq k, \beta \in\left(M^{T}\right)^{-1} \mathbb{Z}^{s} \backslash \mathbb{Z}^{s}, \\
\frac{(-i D)^{\mu}}{\mu !} \hat{y}(0)=e_{\mu}^{T}, \quad|\mu| \leq r .
\end{gathered}
$$

In fact, we can rewrite (3.2) and (3.3) in the time domain (see [10]). First, note that (3.2) and (3.3) are equivalent to

$$
\begin{aligned}
D^{\mu}\left[\hat{y}(\cdot) \hat{\mathbf{a}}\left(\left(M^{T}\right)^{-1} \cdot\right)\right](0) & =D^{\mu}\left[\hat{y}\left(\left(M^{T}\right)^{-1} \cdot\right)\right](0), \quad|\mu| \leq k, \\
D^{\mu}\left[\hat{y}(\cdot) \hat{\mathbf{a}}\left(\left(M^{T}\right)^{-1} \cdot\right)\right](2 \pi \beta) & =0, \quad|\mu| \leq k, \beta \in \mathbb{Z}^{s} \backslash M^{T} \mathbb{Z}^{s} .
\end{aligned}
$$

Let $\Omega_{M}$ denote a complete set of representatives of the quotient group $\mathbb{Z}^{s} / M \mathbb{Z}^{s}$ and define

$$
J_{\varepsilon}^{\mathbf{a}}(\mu):=\sum_{\beta \in \mathbb{Z}^{s}} \mathbf{a}(\varepsilon+M \beta)\left(M^{-1} \varepsilon+\beta\right)^{\mu} / \mu !, \quad \varepsilon \in \Omega_{M} .
$$

It is shown in [10, 13] that (3.5) and (3.6) are equivalent to

$$
\begin{aligned}
& \sum_{0 \leq \nu \leq \mu}(-1)^{|\nu|} \frac{(-i D)^{\mu-\nu} \hat{y}(0)}{(\mu-\nu) !} J_{\varepsilon}^{\mathbf{a}}(\nu) \\
& \quad=\sum_{\nu \in \Lambda_{k}} \mathcal{S}\left(M^{-1}, \Lambda_{k}\right)_{\mu, \nu} \frac{(-i D)^{\nu} \hat{y}(0)}{\nu !}, \quad \forall \mu \in \Lambda_{k}, \quad \varepsilon \in \Omega_{M} .
\end{aligned}
$$

In solving the system of algebraic equations (3.1), 3.7) and (3.4), it may seem at first glance that there is an infinite number of variables since $y$ is a finitely supported sequence (of vectors) with an unknown support size. However, if one takes a closer look at (3.7), one sees that the only variables in the algebraic equations are the entries of $\mathbf{a}(\alpha) \in \mathbb{R}^{m \times m}, \alpha \in \operatorname{supp}(\mathbf{a})$, and those of $Y_{\nu}:=(-i D)^{\nu} \hat{y}(0) / \nu ! \in \mathbb{C}^{1 \times m}, r<|\nu| \leq k$. Thus we do have a system with a finite number of equations and unknowns which one can attempt to solve using symbolic algebraic solvers available in software packages such as MAPLE.

When one is interested only in masks with real entries, then it is enough to assume that $Y_{\nu} \in \mathbb{R}^{1 \times m}$. For a real mask a which also satisfies $\nu_{\infty}(\mathbf{a}, M)$ $>r$, if it satisfies (3.7) with a set of complex vectors $Y_{\nu}$, then it is obvious that $\mathbf{a}$ also satisfies (3.7) with $Y_{\nu}$ replaced by $\operatorname{Re}\left(Y_{\nu}\right)$ or $\operatorname{Im}\left(Y_{\nu}\right)$. Thus, by comments after the proof of Theorem [2.2, we have $\operatorname{Re}\left(Y_{\nu}\right)=c \times \operatorname{Im}\left(Y_{\nu}\right)$ for a real scalar $c$. This implies that looking only for real solutions for $Y_{\nu}$ will not essentially yield a fewer number of solutions than by looking for general complex solutions for $Y_{\nu}$.

We summarize the system of algebraic system to be solved: 


Variables:
\[ \mathbf{a}(\alpha) \in \mathbb{R}^{m \times m}, \alpha \in \operatorname{supp}(\mathbf{a}), \quad Y_{\nu} \in \mathbb{R}^{1 \times m}, r<|\nu| \leq k \]
Equations:
$(3.8) \quad \mathbf{a}(E \alpha)=\mathcal{S}\left(M^{-1} E M, \Lambda_{r}\right) \mathbf{a}(\alpha) \mathcal{S}\left(E^{-1}, \Lambda_{r}\right), \quad \forall \alpha \in \mathbb{Z}^{s}, E \in G, \quad Y_{\mu}=e_{\mu}^{T}, \quad \forall|\mu| \leq r$,
$(3.9) \quad \sum_{0 \leq \nu \leq \mu}(-1)^{|\nu|} Y_{\mu-\nu} J_{\varepsilon}^{\mathbf{a}}(\nu)=\sum_{\nu \in \Lambda_{k}} \mathcal{S}\left(M^{-1}, \Lambda_{k}\right)_{\mu, \nu} Y_{\nu}, \quad \forall \mu \in \Lambda_{k}, \quad \varepsilon \in \Omega_{M}$.

(iv) If multiple solutions are found, optimize over the solution space the following lower bound for $\nu_{\infty}(\mathbf{a}, M)$ [13, 12, 18]: $\nu_{\infty}(\mathbf{a}, M) \geq \nu_{2}(\mathbf{a}, M)-s / 2=$

$$
-\log _{\rho(M)} \sqrt{\rho_{k}}-s / 2=: \gamma(\mathbf{a}),
$$

where $\rho_{k}$ can be calculated by the following procedure:

Let

$$
b(\alpha):=\sum_{\beta} \overline{\mathbf{a}(\beta)} \otimes \mathbf{a}(\alpha+\beta) /|\operatorname{det}(M)|
$$

and

$$
K:=\mathbb{Z}^{s} \bigcap \sum_{n=1}^{\infty} M^{-n}(\operatorname{supp} b) .
$$

Define the size $m^{2}|K|$ matrix $F:=(b(M \alpha-\beta))_{\alpha, \beta \in K}$. Denote the eigenvalues of $M$ by $\sigma=\left(\sigma_{1}, \ldots, \sigma_{s}\right)$. Let $J:=\sum_{\alpha} a(\alpha) /|\operatorname{det} M|$, and $E_{k}=$ $\left\{\lambda \overline{\sigma^{-\mu}}, \bar{\lambda} \sigma^{-\mu}:|\mu| \leq k, \lambda \in \operatorname{spec}(J) \backslash\{1\}\right\} \cup\left\{\sigma^{-\mu}:|\mu| \leq 2 k+1\right\}$. Then

$$
\rho_{k}:=\max \left\{|\nu|: \nu \in \operatorname{spec}(F) \backslash E_{k}\right\} .
$$

(v) If a mask a satisfying $\gamma(\mathbf{a})>r$ is found, then, by Theorem $2.2 S_{\mathbf{a}, M}$ is a Hermite type subdivision scheme and its associated $\phi_{\mathbf{a}, M}$, by results in, e.g., [13, has the following smoothness property:

$$
\phi_{\mathbf{a}, M} \in\left[C^{\sigma}\left(\mathbb{R}^{s}\right)\right]^{m \times 1} \cap\left[W_{2}^{\sigma+s / 2}\left(\mathbb{R}^{s}\right)\right]^{m \times 1}, \quad \forall \sigma<\gamma(\mathbf{a}) .
$$

We had implemented a solver based on the above method using a mixture of symbolic and numerical computational tools. We now present some results obtained using our software, and we discuss along the way questions stimulated by these examples. As in [16], we consider the following cases:

- $G=\{[1],[-1]\}, M=[2]$.

- the hexagonal symmetry group (a.k.a. dihedral group $D_{6}$ ) with respect to $M=2 I_{2}$ :

$$
D_{6}=\left\{ \pm\left[\begin{array}{ll}
1 & 0 \\
0 & 1
\end{array}\right], \pm\left[\begin{array}{ll}
0 & -1 \\
1 & -1
\end{array}\right], \pm\left[\begin{array}{ll}
-1 & 1 \\
-1 & 0
\end{array}\right], \pm\left[\begin{array}{ll}
0 & 1 \\
1 & 0
\end{array}\right], \pm\left[\begin{array}{ll}
1 & -1 \\
0 & -1
\end{array}\right], \pm\left[\begin{array}{ll}
-1 & 0 \\
-1 & 1
\end{array}\right]\right\}
$$

- the square symmetry group (a.k.a. dihedral group $D_{4}$ ) with respect to $M=2 I_{2}$ :

$$
D_{4}=\left\{ \pm\left[\begin{array}{ll}
1 & 0 \\
0 & 1
\end{array}\right], \pm\left[\begin{array}{cc}
0 & -1 \\
1 & 0
\end{array}\right], \pm\left[\begin{array}{cc}
1 & 0 \\
0 & -1
\end{array}\right], \pm\left[\begin{array}{ll}
0 & 1 \\
1 & 0
\end{array}\right]\right\}
$$

- $D_{6}$ with respect to $M=M_{\sqrt{3}}:=\left[\begin{array}{ll}1 & -2 \\ 2 & -1\end{array}\right]$.

- $D_{4}$ with respect to $M=M_{\text {Quincunx }}:=\left[\begin{array}{cc}1 & 1 \\ 1 & -1\end{array}\right]$. 
Note that (2.10) is satisfied in each case.

3.1. $G=\{[1],[-1]\}, M=[2], \operatorname{supp}(\mathbf{a})=[-2,2], r=1, k=7$. In this case, the following two distinct solutions which satisfy the sum rules of order 8 (i.e., $k=7$ ) are found:

$$
\begin{gathered}
\mathbf{a}^{1}(0)=\left[\begin{array}{cc}
63 / 64 & 0 \\
0 & 3 / 8
\end{array}\right], \quad \mathbf{a}^{1}(1)=\left[\begin{array}{cc}
1 / 2 & -15 / 16 \\
1 / 16 & -7 / 32
\end{array}\right], \\
\mathbf{a}^{1}(2)=\left[\begin{array}{cc}
1 / 128 & 0 \\
-7 / 256 & 1 / 16
\end{array}\right], \quad \mathbf{a}^{2}(0)=\left[\begin{array}{cc}
41 / 48 & 0 \\
0 & 19 / 96
\end{array}\right], \\
\mathbf{a}^{2}(1)=\left[\begin{array}{cc}
1 / 2 & -7 / 12 \\
5 / 56 & -1 / 24
\end{array}\right], \mathbf{a}^{2}(2)=\left[\begin{array}{cc}
7 / 96 & -77 / 384 \\
25 / 1344 & -19 / 384
\end{array}\right], \\
\mathbf{a}^{i}(-\alpha)=N^{-1} \mathbf{a}^{i}(\alpha) N,
\end{gathered}
$$

where $N=\operatorname{diag}([1,-1])$.

Using the procedure explained in steps (iv) $-(\mathrm{v})$ above, we found that

$$
\phi_{\mathbf{a}^{1}} \in\left[W_{2}^{\sigma}(\mathbb{R})\right]^{2 \times 1} \cap\left[C^{\sigma-0.5}(\mathbb{R})\right]^{2 \times 1}, \quad \forall \sigma<4.5,
$$

and

$$
\phi_{\mathbf{a}^{2}} \in\left[W_{2}^{\sigma}(\mathbb{R})\right]^{2 \times 1} \cap\left[C^{\sigma-0.5}(\mathbb{R})\right]^{2 \times 1}, \quad \forall \sigma<6.5 .
$$

From computation both schemes seem to produce spline functions of piecewise degree 7 polynomials. See Figures 35 for graphical evidence.

Discussion. It is well known that in the scalar case $(r=0)$ if one solves for the mask supported at $[-2,2]$ satisfying the highest possible sum rule order, then one gets the subdivision mask $a=[\cdots 0,1,4,6,4,1,0 \cdots] / 8$, which satisfies the sum rules of order 4 and $\phi_{a}$ is the cubic $B$-spline. Since $a^{1}$ and $a^{2}$ are found using a similar principle and they both occur to generate spline functions, we question how to characterize such spline functions.

It is known that multiple-knot spline functions, i.e., functions in

$$
\mathcal{S}_{k, m}:=\left\{f \in C^{k-m}(\mathbb{R}):\left.f\right|_{(\alpha, \alpha+1)} \in \Pi_{k}, \forall \alpha \in \mathbb{Z}\right\},
$$

can be computed using vector subdivision schemes; see, e.g., [25, 8]. Taking a slightly different approach, the authors observe that, based on the Deboor algorithm [3, $\mathcal{S}_{k, m}$ can be generated by a subdivision scheme of Lagrange type of multiplicity $m$, defined as follows:

Definition 3.1. A subdivision scheme $S:=S_{\mathrm{a}, M}$ is of Lagrange type of multiplicity $m$ if for any initial sequence $v \in\left[l^{0}\left(\mathbb{Z}^{s}\right)\right]^{1 \times m}$ there exists $f_{v} \in C\left(\mathbb{R}^{s}\right)$ such that $\lim _{n \rightarrow \infty} \max _{\alpha \in \mathbb{Z}^{s}} \max _{1 \leq i \leq m}\left|f_{v}\left(M^{-n} \alpha\right)-\left(S^{n} v\right)(\alpha)_{i}\right|=0$, and, moreover, $f_{v} \neq 0$ for some $v \neq 0$.

For example, the spline space $\mathcal{S}_{7,2}$ is generated by $\phi_{\mathbf{a}^{3}}$ where $\operatorname{supp}\left(\mathbf{a}^{3}\right)=[-2,2]$ and

$$
\begin{gathered}
\mathbf{a}^{3}(0)=\left[\begin{array}{cc}
\frac{101}{192} & \frac{21}{64} \\
\frac{21}{64} & \frac{101}{192}
\end{array}\right], \quad \mathbf{a}^{3}(1)=\left[\begin{array}{cc}
\frac{5}{32} & \frac{1}{32} \\
\frac{49}{96} & \frac{29}{96}
\end{array}\right], \\
\mathbf{a}^{3}(2)=\left[\begin{array}{cc}
\frac{1}{128} & 0 \\
\frac{47}{384} & \frac{1}{64}
\end{array}\right], \quad \mathbf{a}^{3}(-\alpha)=J \mathbf{a}^{3}(\alpha) J, \quad J=\left[\begin{array}{ll}
0 & 1 \\
1 & 0
\end{array}\right] .
\end{gathered}
$$



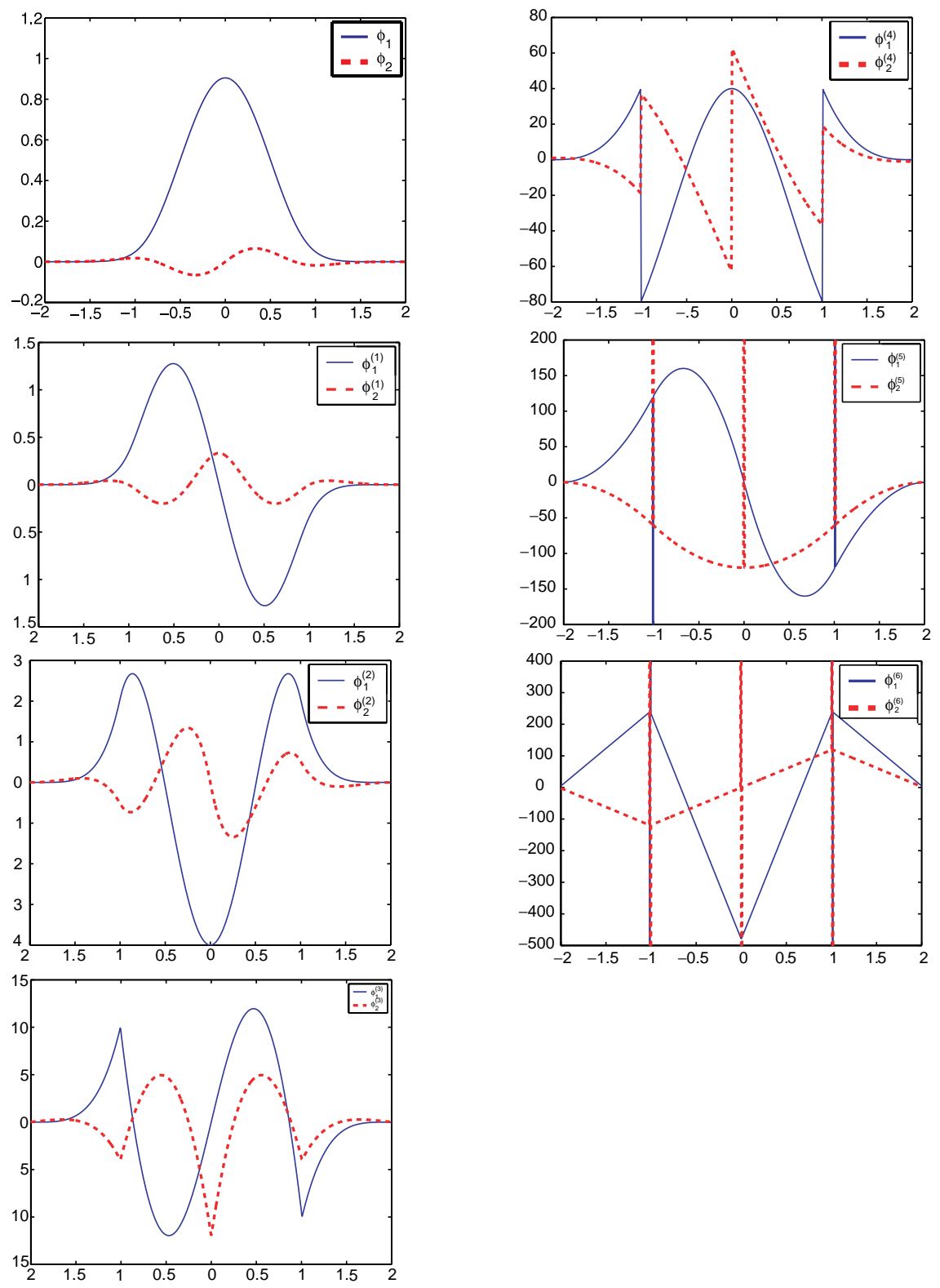

Figure 3. $\phi_{\mathbf{a}^{1}}$ and their derivatives: (from top to bottom, left then right) $\left(\phi_{\mathbf{a}^{1}}\right)_{1}^{(l)}$ (solid blue line), $\left(\phi_{\mathbf{a}^{1}}\right)_{2}^{(l)}$ (dashed red line) for $l=0,1, \ldots, 6$. It is evident from these plots that $\phi_{\mathbf{a}^{1}}$ consists of piecewise degree 7 polynomial functions with $C^{3}$ knots at the integers. While the functions are not $C^{4}$ at the knots, the plots suggest that $\phi_{\mathbf{a}^{1}}^{(5)}$ and $\phi_{\mathbf{a}^{1}}^{(6)}$ are continuous, i.e., $\phi_{\mathbf{a}^{1}}^{(l)}(\alpha+)=\phi_{\mathbf{a}^{1}}^{(l)}(\alpha-)$ for $l=5,6$ and $\alpha \in \mathbb{Z}$. 

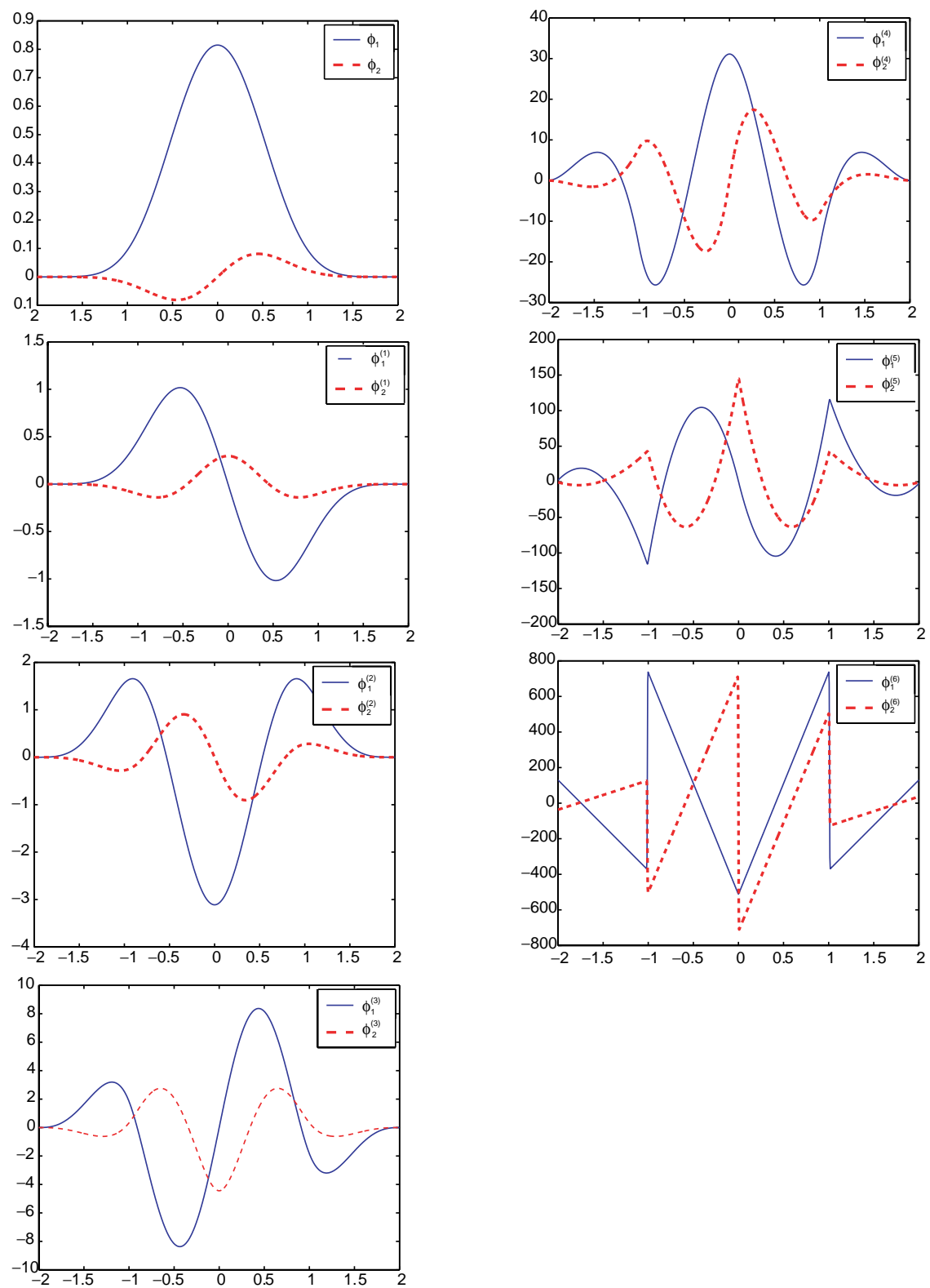

Figure 4. $\phi_{\mathbf{a}^{2}}$ and their derivatives: (from top to bottom, left then right) $\left(\phi_{\mathbf{a}^{2}}\right)_{1}^{(l)}$ (solid blue line), $\left(\phi_{\mathbf{a}^{2}}\right)_{2}^{(l)}$ (dashed red line) for $l=0,1, \ldots, 6$. It is evident from these plots that $\phi_{\mathbf{a}^{2}}$ consists of piecewise degree 7 polynomials with $C^{5}$ knots at the integers.

Notice that there exists a linear transform $T_{\text {Lagrange } \rightarrow \text { Hermite, }}$, independent of the $x_{0}$ and $h>0$ below, such that for any $f \in \Pi_{r}$ we have

$$
\begin{aligned}
& {\left[f\left(x_{0}\right), h f^{\prime}\left(x_{0}\right), \ldots, h^{r} f^{(r)}\left(x_{0}\right)\right]^{T}} \\
& \quad=T_{\text {Lagrange } \rightarrow \text { Hermite }} \times\left[f\left(x_{0}\right), f\left(x_{0}+h\right), \ldots, f\left(x_{0}+r h\right)\right]^{T} .
\end{aligned}
$$


By virtue of (3.14), we expect that under appropriate technical assumptions on a Lagrange type subdivision scheme, it can be transformed into a Hermite type scheme by applying a constant similarity transform on the mask. A detailed formulation and analysis on this matter is out of the scope of this article, and we view it as a heuristics at this point. Using this heuristics, we discover that $\mathbf{a}^{2}(\alpha)=T \mathbf{a}^{3}(\alpha) T^{-1}$, where $T=\left[\begin{array}{cc}\frac{1}{2} & \frac{1}{2} \\ -\frac{1}{7} & \frac{1}{7}\end{array}\right]$. Consequently, $\phi_{\mathbf{a}^{2}}=T \phi_{\mathbf{a}^{3}}$ and

$$
\operatorname{span}\left\{\phi_{\mathbf{a}^{2}}(\cdot-\alpha): \alpha \in \mathbb{Z}\right\}=\operatorname{span}\left\{\phi_{\mathbf{a}^{3}}(\cdot-\alpha): \alpha \in \mathbb{Z}\right\}=\mathcal{S}_{7,2} \cdot
$$

On the other hand, Figure 3 suggests that

$$
\operatorname{span}\left\{\phi_{\mathbf{a}^{1}}(\cdot-\alpha): \alpha \in \mathbb{Z}\right\} \varsubsetneqq \mathcal{S}_{7,4} \cdot
$$

In fact, from the classical work on splines by Schoenberg, the shift-invariant space $\mathcal{S}_{7,4}$ requires four linearly independent functions to generate. So it is impossible that equality holds above. Moreover, Figure 3] strongly suggests that $\phi_{\mathbf{a}^{1}}$, while not four times continuously differentiable, has the property that $\phi_{\mathbf{a}^{1}}^{(l)}(\alpha+)=\phi_{\mathbf{a}^{1}}^{(l)}(\alpha-)$ for $l=5,6$ and $\alpha \in \mathbb{Z}$, but a general function in $\mathcal{S}_{7,4}$ does not satisfy this property. All these suggest that $\phi_{\mathbf{a}^{1}}$ generates a proper subspace of $\mathcal{S}_{7,4}$.

3.2. $G=D_{4}, M=2 I_{2}, \operatorname{supp}(\mathbf{a})=[-2,2]^{2}, r=1, k=6$. One family of solutions obtained from our solver is

$$
\begin{gathered}
\mathbf{a}(0,0)=\operatorname{diag}\left[\frac{9}{16},-8 t+\frac{9}{64},-8 t+\frac{9}{64}\right], \\
\mathbf{a}(2,0)=\left[\begin{array}{ccc}
\frac{15}{128} & -\frac{15}{128}-30 t & 0 \\
\frac{39}{128} & -\frac{3}{128}-8 t & 0 \\
0 & 0 & 4 t
\end{array}\right], \\
\mathbf{a}(1,1)=\left[\begin{array}{ccc}
\frac{1}{4} & -\frac{15}{64} & -\frac{15}{64} \\
\frac{3}{80} & \frac{1}{128} & -\frac{3}{64} \\
\frac{3}{80} & -\frac{3}{64} & \frac{1}{128}
\end{array}\right], \\
\mathbf{a}(1,0)=\left[\begin{array}{ccc}
\frac{51}{128} & -\frac{15}{64} & 0 \\
\frac{39}{640} & \frac{3}{64} & 0 \\
0 & 0 & \frac{9}{128}
\end{array}\right] \\
\mathbf{a}(1,2)=\left[\begin{array}{ccc}
\frac{13}{256} & -\frac{15}{128} & -\frac{15}{128} \\
\frac{9}{1280} & -\frac{1}{64} & -\frac{3}{128} \\
\frac{33}{2560} & -\frac{9}{256} & -\frac{7}{256}
\end{array}\right] .
\end{gathered}
$$

In Figure 5(a) we plot $\nu_{2}(\mathbf{a}, M)$ for $t \in[0.001,0.01]$. It shows that $\nu_{2}(\mathbf{a}, M)=5$ on a subinterval. We also obtain other multiple-parameter families of solutions which satisfy the sum rules of order 7 ; however, by optimizing the objective $\nu_{2}(\mathbf{a}, M)$ function over these parameters, we did not discover schemes with $L^{2}$ Sobolev smoothness higher than 5 .

\footnotetext{
${ }^{1}$ For instance, it is clearly necessary that $m=\# \Lambda_{r}$ and that the limit function $f$ be $r$-th times continuously differentiable. But even under these assumptions, there are still lurking possibilities such as that the Lagrange type scheme is made up of $m$ copies of a single scalar subdivision scheme. The condition $\nu_{\infty}(\mathbf{a}, M)>r$ would avoid the latter and guarantee $f \in C^{r}$ at the same time.
} 

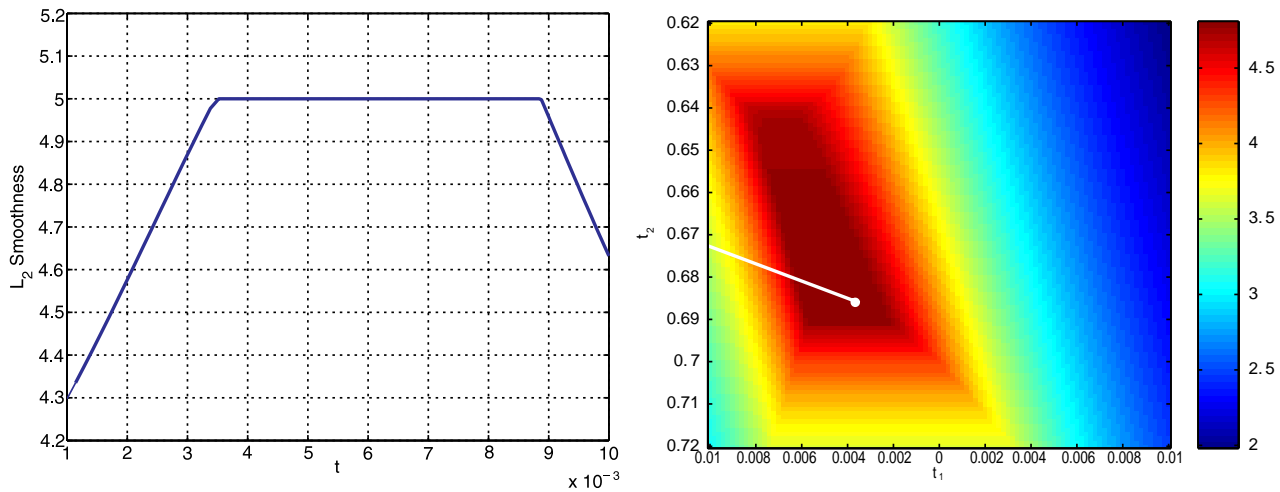

Figure 5. (a) $\nu_{2}(\mathbf{a}, M)$ for $t \in[0.001,0.01]$, where $\mathbf{a}$ is the 1parameter mask in Section 3.2. (b) color map of $\nu_{2}(\mathbf{a}, M)$ for $\left(t_{1}, t_{2}\right) \in[-0.01,0.01] \times[-0.72,-0.62]$, where $\mathbf{a}$ is the 2-parameter mask in Section [3.3, the white dot is the computed optimizer $\left(t_{1}, t_{2}\right) \approx(-0.003667,-0.6860)$.

3.3. $G=D_{6}, M=2 I_{2}, \operatorname{supp}(\mathbf{a}) \subset[-2,2]^{2}, r=1, k=4$. We consider the smallest $D_{6}$-symmetric supports which contain $\{(0,0),(0,1),(0,2),(1,2)\}$, i.e., $\operatorname{supp}(\mathbf{a})=$ $\{(0,0), \pm(0,2), \pm(2,0), \pm(2,2), \pm(1,0), \pm(1,2), \pm(0,1), \pm(2,1), \pm(1,1), \pm(1,-1)$, $|\operatorname{supp}(\mathbf{a})|=19$. In this case, our solver again gives us several sets of multi-parameter families of solutions. One such family is

$$
\begin{gathered}
\mathbf{a}(0,0)=\operatorname{diag}\left[\frac{47}{32}+\frac{9 t_{2}}{8},-1-24 t_{1}-\frac{3 t_{2}}{2},-1-24 t_{1}-\frac{3 t_{2}}{2}\right] \\
\mathbf{a}(2,0)=\left[\begin{array}{ccc}
-\frac{5}{64}-\frac{3}{16} t_{2} & -\frac{1}{2}-\frac{1}{2} t_{2} & \frac{1}{4}+\frac{1}{4} t_{2} \\
-\frac{1}{32}-\frac{1}{16} t_{2} & -\frac{5}{16}-4 t_{1}-\frac{3}{8} t_{2} & \frac{5}{16}+8 t_{1}+\frac{3}{8} t_{2} \\
0 & 0 & \frac{5}{16}+12 t_{1}+\frac{3}{8} t_{2}
\end{array}\right], \\
\mathbf{a}(1,1)=\left[\begin{array}{ccc}
\frac{21}{32}+\frac{3}{8} t_{2} & -\frac{3}{4}-\frac{3}{4} t_{2} & -\frac{3}{4}-\frac{3}{4} t_{2} \\
\frac{3}{16}+\frac{3}{16} t_{2} & -\frac{5}{16}-\frac{1}{2} t_{2} & -\frac{1}{8}-\frac{1}{8} t_{2} \\
\frac{3}{16}+\frac{3}{16} t_{2} & -\frac{1}{8}-\frac{1}{8} t_{2} & -\frac{5}{16}-\frac{1}{2} t_{2}
\end{array}\right], \\
\mathbf{a}(1,-1)=\left[\begin{array}{ccc}
-\frac{5}{32}-\frac{3}{8} t_{2} & -\frac{3}{4}-\frac{3}{4} t_{2} & \frac{3}{4}+\frac{3}{4} t_{2} \\
-\frac{1}{32}-\frac{1}{16} t_{2} & -\frac{3}{16}-\frac{1}{4} t_{2} & \frac{1}{8}+\frac{1}{8} t_{2} \\
\frac{1}{32}+\frac{1}{16} t_{2} & \frac{1}{8}+\frac{1}{8} t_{2} & -\frac{3}{16}-\frac{1}{4} t_{2}
\end{array}\right] .
\end{gathered}
$$

Using the optimization code developed in [14], we found that the empirical optimal $L^{2}$ Sobolev smoothness among this family is 4.8103 , and the optimizing parameter values $\left(t_{1}, t_{2}\right) \approx(-0.003667,-0.6860)$ seem to be unique. Figure 5 (b) shows a color map of $\nu_{2}(\mathbf{a}, M)$ for $\left(t_{1}, t_{2}\right) \in[-0.01,0.01] \times[-0.72,-0.62]$. Another set of 
solutions obtained by our symbolic solver is

$$
\begin{gathered}
\mathbf{a}(0,0)=\operatorname{diag}\left[6 t_{3}+\frac{11}{32}, \frac{1}{2}-24 t_{2}-24 t_{1}, \frac{1}{2}-24 t_{2}-24 t_{1}\right], \\
\mathbf{a}(2,0)=\left[\begin{array}{ccc}
-t_{3}+\frac{7}{64} & -8 t_{2} & 4 t_{2} \\
-\frac{1}{3} t_{3}+\frac{1}{32} & -4 t_{1}-\frac{1}{3} t_{3}-5 t_{2}+\frac{1}{16} & \frac{1}{3} t_{3}+5 t_{2}-\frac{1}{16}+8 t_{1} \\
0 & 0 & \frac{1}{3} t_{3}+5 t_{2}-\frac{1}{16}+12 t_{1}
\end{array}\right], \\
\mathbf{a}(1,1)=\left[\begin{array}{ccc}
2 t_{3}+\frac{9}{32} & -4 t_{3} & -4 t_{3} \\
t_{3} & -\frac{8}{3} t_{3}+\frac{3}{16} & -\frac{2}{3} t_{3} \\
t_{3} & -\frac{2}{3} t_{3} & -\frac{8}{3} t_{3}+\frac{3}{16}
\end{array}\right] \\
\mathbf{a}(1,-1)=\left[\begin{array}{ccc}
-2 t_{3}+\frac{7}{32} & -4 t_{3} & 4 t_{3} \\
-\frac{1}{3} t_{3}+\frac{1}{32} & -\frac{4}{3} t_{3}+\frac{1}{16} & \frac{2}{3} t_{3} \\
\frac{1}{3} t_{3}-\frac{1}{32} & \frac{2}{3} t_{3} & -\frac{4}{3} t_{3}+\frac{1}{16}
\end{array}\right] .
\end{gathered}
$$

Optimizing $\nu_{2}(\mathbf{a}, M)$ over this family gives the optimal $L^{2}$ Sobolev smoothness 4.8188 , attained at parameter values $\left(t_{1}, t_{2}, t_{3}\right)=(-0.0043,0.0191,0.0603)$.

3.4. $G=D_{6}, M=M_{\sqrt{3}}, \operatorname{supp}(\mathbf{a}) \subset[-2,2]^{2}, r=1, k=6$. We consider the smallest $D_{6}$-symmetric supports which contain $\{(0,0),(0,1),(0,2),(1,2)\}$; we have $|\operatorname{supp}(\mathbf{a})|=19$.

A unique solution which satisfies the sum rules of order 7 is found using our solver, with the following rather peculiar form:

$$
\begin{gathered}
\mathbf{a}(0,0)=\left[\begin{array}{ccc}
-\frac{11}{6} \theta+\frac{119}{108} & 0 & 0 \\
0 & -\frac{3}{2} \theta^{2}+\frac{169}{108} \theta-\frac{31}{81} & 3 \theta^{2}-\frac{169}{54} \theta+\frac{62}{81} \\
0 & -3 \theta^{2}+\frac{169}{54} \theta-\frac{62}{81} & -\frac{169}{108} \theta+\frac{3}{2} \theta^{2}+\frac{31}{81}
\end{array}\right], \\
\mathbf{a}(0,2)=\left[\begin{array}{ccc}
\frac{5}{18} \theta-\frac{17}{324} & -\frac{73}{36} \theta+\frac{8}{27}+\frac{9}{2} \theta^{2} & \frac{73}{18} \theta-\frac{16}{27}-9 \theta^{2} \\
\frac{1}{12} \theta-\frac{11}{648} & -\frac{535}{448} \theta+\frac{7}{4} \theta^{2}+\frac{29}{243} & \frac{535}{324} \theta-\frac{7}{2} \theta^{2}-\frac{58}{243} \\
\frac{1}{24} \theta-\frac{11}{1296} & -\frac{413}{648} \theta+\frac{5}{4} \theta^{2}+\frac{22}{243} & \frac{535}{648} \theta-\frac{7}{4} \theta^{2}-\frac{29}{243}
\end{array}\right], \\
\mathbf{a}(0,1)=\left[\begin{array}{ccc}
-\frac{5}{18} \theta+\frac{125}{324} & -\frac{4}{3} \theta+\frac{16}{27} & \frac{8}{3} \theta-\frac{32}{27} \\
-\frac{2}{9} \theta+\frac{1}{9} & \frac{1}{2} \theta^{2}-\frac{277}{324} \theta+\frac{52}{243} & -\theta^{2}+\frac{277}{162} \theta-\frac{104}{243} \\
\frac{1}{18}-\frac{1}{9} \theta & \frac{11}{2} \theta^{2}-\frac{995}{324} \theta+\frac{104}{243} & -\frac{1}{2} \theta^{2}+\frac{277}{324} \theta-\frac{52}{243}
\end{array}\right], \\
\mathbf{a}(1,2)=\left[\begin{array}{ccc}
\frac{11}{36} \theta-\frac{11}{648} & 0 & \frac{9}{2} \theta^{2}+\frac{7}{27}-\frac{97}{36} \theta \\
\frac{5}{72} \theta-\frac{5}{1296} & \frac{79}{81} \theta-\frac{65}{486}-2 \theta^{2} & -\frac{607}{648} \theta+\frac{49}{486}+\frac{7}{4} \theta^{2} \\
0 & \frac{158}{81} \theta-\frac{65}{243}-4 \theta^{2} & -\frac{79}{81} \theta+2 \theta^{2}+\frac{65}{486}
\end{array}\right],
\end{gathered}
$$

where $\theta \approx 0.2587$ is the unique real root of the polynomial $972 \theta^{3}-744 \theta^{2}+197 \theta-18$. By computation, we have $\nu_{2}(\mathbf{a}, M)=5.76867$ and, consequently, $\phi \in C^{4}$. 
In comparison, for the scalar scheme $\mathbf{a}(0,0)=1 / 3, \mathbf{a}(1,2)=1 / 9, \mathbf{a}(0,1)=2 / 9$, $\mathbf{a}(0,2)=1 / 9$ with the same $D_{6}$-symmetric support, $\phi_{\mathbf{a}}$ is a $C^{3}$ (quartic) box-spline. For other scalar schemes under the settings of this section, see [20. Table 3].

3.5. $G=D_{4}, M=M_{\text {Quincunx }}, \operatorname{supp}(\mathbf{a})=[-1,1]^{2}, r=1, k=8$. In the case $G=D_{4}, M=M_{\text {Quincunx }}, \operatorname{supp}(\mathbf{a})=[-1,1]^{2}$ but with $r=0$, the mask $\mathbf{a}(0,0)=$ $1 / 2, \mathbf{a}(1,1)=\mathbf{a}(-1,1)=\mathbf{a}(-1,-1), \mathbf{a}(1,-1)=1 / 8, \mathbf{a}(1,0)=\mathbf{a}(0,1)=\mathbf{a}(-1,0)=$ $\mathbf{a}(0,-1)=1 / 4$ is known to generate a box spline $\phi$ which is a $C^{4}$ piecewise degree 6 polynomial [26, 4]. This box spline is used in [26] for building free-form subdivision surfaces. In fact this mask satisfies the sum rules of order 4 and $\nu_{2}(\mathbf{a}, M)=4$. Due to the linear dependence of the shifts of this box-spline, (3.10) would not give a sharp $L^{2}$ smoothness exponent. However, from the spline property of $\phi$ we have

$$
\phi \in\left[W_{2}^{5.5-\varepsilon}\left(\mathbb{R}^{2}\right)\right]^{3 \times 1} \cap\left[C^{5-\varepsilon}\left(\mathbb{R}^{2}\right)\right]^{3 \times 1}, \quad \forall \varepsilon>0 .
$$

We now consider $r=1$. Using our solver, we found the following unique mask satisfying the sum rules of order 9:

$$
\begin{gathered}
\mathbf{a}(0,0)=\left[\begin{array}{ccc}
\frac{1}{2} & 0 & 0 \\
0 & \frac{1}{16} & \frac{1}{16} \\
0 & \frac{1}{16} & -\frac{1}{16}
\end{array}\right], \quad \mathbf{a}(1,1)=\left[\begin{array}{ccc}
\frac{1}{8} & -\frac{1}{4} & -\frac{1}{4} \\
\frac{1}{32} & -\frac{1}{16} & -\frac{1}{16} \\
0 & \frac{1}{32} & -\frac{1}{32}
\end{array}\right], \\
\mathbf{a}(1,0)=\left[\begin{array}{ccc}
\frac{1}{4} & -\frac{1}{2} & 0 \\
\frac{1}{32} & -\frac{1}{32} & \frac{1}{32} \\
\frac{1}{32} & -\frac{1}{32} & -\frac{1}{32}
\end{array}\right] .
\end{gathered}
$$

By (3.10)-(3.11), we found by computation that $\nu_{2}(\mathbf{a}, M)=8.5$ and therefore,

$$
\phi_{\mathbf{a}} \in\left[W_{2}^{8.5-\varepsilon}\left(\mathbb{R}^{2}\right)\right]^{3 \times 1} \cap\left[C^{7.5-\varepsilon}\left(\mathbb{R}^{2}\right)\right]^{3 \times 1}, \quad \forall \varepsilon>0 .
$$

It is, however, unknown at this point whether the components of $\phi_{\mathbf{a}}$ are spline functions, although by plotting the divided differences of $\phi_{\mathbf{a}}$, we observe empirically that they are piecewise degree 10 polynomials.

\section{Concluding Remarks and open questions}

We have introduced in this article the notion of noninterpolatory Hermite subdivision schemes in a general dimension. Using the strong convergence theory of refinable functions, we have obtained a constructive sufficient condition on the mask of noninterpolatory Hermite subdivision schemes. We have also studied symmetry conditions for such schemes. A number of symmetric bivariate schemes with small supports have been constructed with their regularity exponents computed.

4.1. Connection to interpolatory Hermite schemes. A refinable Hermite interpolant $\phi=\left(\phi_{\mu}\right)_{\mu \in \Lambda_{r}}$ which is also in $C^{k}, k \geq r$, must have an accuracy order of $k+1: \operatorname{span}\left\{g(\cdot-\alpha): \alpha \in \mathbb{Z}^{s}\right\} \supseteq \Pi_{k}$ where $g=\sum_{\alpha \in \mathbb{Z}^{s}} y(\alpha) \phi(\cdot-\alpha)$ for a $y \in\left[l^{0}\left(\mathbb{Z}^{s}\right)\right]^{1 \times \# \Lambda_{r}}$ which satisfies

$$
Y_{\nu}:=\frac{(-i D)^{\nu}}{\nu !} \hat{y}(0)=\left\{\begin{array}{cc}
e_{\nu}^{T}, & |\nu| \leq r \\
0, & r<|\nu| \leq k
\end{array}\right.
$$


By [16, Proposition 2.1], the mask of $\phi$ satisfies

$$
\mathbf{a}(M \alpha)=\delta_{\alpha, 0} \mathcal{S}\left(M^{-1}, \Lambda_{r}\right) .
$$

Since the $Y_{\nu}, r<|\nu| \leq k$, are no longer variables, (4.1) and (4.2) turn the generally nonlinear system of equations (3.8) and (3.9) into a linear system of equations, and the latter can be shown to be identical to the (seemingly different) linear system of equations used in [16, Section 3] for constructing interpolatory Hermite subdivision schemes.

4.2. Dual noninterpolatory Hermite schemes. As it turns out, the symmetry conditions studied in Section 2.1 are related to what the CAGD community calls primal subdivision schemes or vertex-based schemes. It is possible to construct dual (a.k.a. face-based) noninterpolatory Hermite subdivision schemes by formulating a different symmetry condition; we plan to report results in this direction elsewhere [15].

4.3. Open questions. Several analysis questions pertaining to Hermite type subdivision schemes are open at the time of the writing of this article:

(1) Is the sufficient condition in Theorem 2.2 also necessary?

This question has been answered negatively in [15].

(2) If $\mathbf{a}$ is the mask of a Hermite type subdivision scheme and $\phi$ is the associated refinable function vector, can we guarantee that $\phi$ cannot satisfy another refinement equation

$$
\phi(x)=\sum_{\beta} \mathbf{b}(\beta) \phi(M x-\beta)
$$

with $\mathbf{b} \neq \mathbf{a}$ ?

We are interested in this uniqueness property because if it holds, then, by the discussion after the proof of Proposition 2.3, the three symmetry conditions (1)-(3) in Proposition 2.3 are equivalent.

In the scalar case $r=0$, the uniqueness property is clear: since $\hat{\phi}(\omega)=$ $\hat{\mathbf{a}}\left(M^{-T} \omega\right) \hat{\phi}\left(M^{-T} \omega\right)$,

$$
\hat{\mathbf{a}}(\omega)=\sum_{\alpha} \mathbf{a}(\alpha) e^{-i\langle\omega, \alpha\rangle} /|\operatorname{det} M|,
$$

and $\hat{\phi}(\omega) \neq 0$ except on a countable number of points, it follows that $\hat{\mathbf{a}}(\omega)=\hat{\phi}\left(M^{T} \omega\right) / \hat{\phi}(\omega)$ holds at an infinite number of points $\omega$. But the trigonometrical polynomial â is uniquely determined by its values at a finite number of points; thus the uniqueness property holds.

In general, consider $\hat{\mathbf{a}}(\omega) \hat{\phi}(\omega+2 \pi \beta)=\hat{\phi}\left(M^{T}(\omega+2 \pi \beta)\right.$. Then we have the uniqueness property if

$\operatorname{span}\left\{\hat{\phi}(\omega+2 \pi \beta): \beta \in \mathbb{Z}^{s}\right\}=\mathbb{C}^{\# \Lambda_{r}}$ for an infinite number of $\omega \in \mathbb{R}^{s}$.

One may find condition (4.3) somewhat familiar since it is known [19] that stability of the integer shifts of $\phi$ is equivalent to the condition

$$
\operatorname{span}\left\{\hat{\phi}(\omega+2 \pi \beta): \beta \in \mathbb{Z}^{s}\right\}=\mathbb{C}^{\# \Lambda_{r}} \text { for all } \omega \in \mathbb{R}^{s},
$$

which is clearly stronger than (4.3).

(3) Is it the case that the solutions of the system (3.8)-(3.9) contain all the possible Hermite type schemes with the given constraints? 


\section{REFERENCES}

[1] E. Catmull and J. Clark. Recursive generated B-spline surfaces on arbitrary topological meshes. Comp. Aid. Geom. Des., 10(6):350-355, 1978.

[2] A. Cohen, N. Dyn, and D. Levin. Matrix subdivision scheme. Unpublished manuscript, avail-

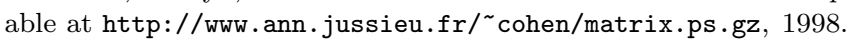

[3] C. de Boor. A Practical Guide to Splines. Number 27 in Applied Mathematical Sciences. Springer-Verlag, New York, 1978.

[4] C. de Boor, K. Höllig, and S. Riemenschneider. Box Splines. Springer-Verlag, 1993. MR 94k:65004

[5] D. L. Donoho, N. Dyn, D. Levin, and T. P.-Y. Yu. Smooth multiwavelet duals of Alpert bases by moment-interpolating refinement. Appl. Comput. Harmon. Anal., 9(2):166-203, 2000. MR 2001j:42029

[6] D. Doo and M. Sabin. Analysis of the behavoir of recursive division surfaces near extraordinary points. Comp. Aid. Geom. Des., 10(6):356-360, 1978.

[7] N. Dyn and D. Levin. Analysis of Hermite-interpolatory subdivision schemes. In S. Dubuc and G. Deslauriers, editors, Spline Functions and the Theory of Wavelets, pages 105-113, 1999. CRM (Centre de Recherches Mathématiques, Université de Montréal) Proceedings \& Lectures Notes, Volume 18. MR99j:42040

[8] N. Dyn and D. Levin. Subdivision schemes in geometric modelling. Acta Numerica, 11:73-144, 2002. MR 2004g:65017

[9] N. Dyn, D. Levin, and J. A. Gregory. A butterfly subdivision scheme for surface interpolation with tension control. ACM Transaction on Graphics, 9(2), April 1990.

[10] B. Han. Approximation properties and construction of Hermite interpolants and biorthogonal multiwavelets. J. Approx. Theory, 110(1):18-53, 2001. MR2002e:41008

[11] B. Han. Symmetry property and construction of wavelets with a general dilation matrix. Linear Algebra and its Applications, 353:207-225, 2002. MR2003g:42059

[12] B. Han. Computing the smoothness exponent of a symmetric multivariate refinable function. SIAM J. Matrix Anal. and Appl., 24(3):693-714, 2003. MR2004b:42078

[13] B. Han. Vector cascade algorithms and refinable function vectors in Sobolev spaces. Journal of Approximation Theory, 124(1):44-88, 2003. MR:2004h:42034

[14] B. Han, M. Overton, and T. P.-Y. Yu. Design of Hermite subdivision schemes aided by spectral radius optimization. SIAM Journal on Scientific Computing, 25(2):643-656, 2003.

[15] B. Han and T. P.-Y. Yu. Face-based Hermite subdivision schemes. Preprint, available at http://www.rpi.edu/ yut/Papers/dual.pdf, September 2003.

[16] B. Han, T. P.-Y. Yu, and B. Piper. Multivariate refinable Hermite interpolants. Mathematics of Computation, 2003. To appear. Preprint available at http://www.rpi.edu/ yut/hyp.pdf.

[17] L. Hervé. Multiresolution analysis of multiplicity $d$ : applications to dyadic interpolation. Appl. Comput. Harmon. Anal., 1:299-315, 1994. MR97a:42026

[18] R. Q. Jia and Q. T. Jiang. Spectral analysis of the transition operator and its applications to smoothness analysis of wavelets. SIAM J. Matrix Anal. and Appl., 24(4):1071-1109, 2003. MR 2004h:42043

[19] R. Q. Jia and C. A. Micchelli. On linear independence of integer translates of a finite number of functions. Proc. Edinburgh Math. Soc., pages 69-85, 1992. MR94e:41044

[20] Q. Jiang and P. Oswald. Triangular $\sqrt{3}$-subdivision schemes: the regular case. J. Comput. Appl. Math., 156(1):47-75, 2003. MF2004f:65018

[21] L. Kobbelt. $\sqrt{3}$ subdivision. Computer Graphics Proceedings (SIGGRAPH 2000), 2000.

[22] L. Kobbelt, T. Hesse, H. Prautzsch, and K. Schweizerhof. Interpolatory subdivision on open quadrilateral nets with arbitrary topology. In Proceedings of Eurographics '96, Computer Graphics Forum 15, pages 409-420, 1996.

[23] C. T. Loop. Smooth subdivision surfaces based on triangles. Master's thesis, Department of Mathematics, University of Utah, 1987.

[24] J. L. Merrien. A family of Hermite interpolants by bisection algorithms. Numerical Algorithms, 2:187-200, 1992. MR93b:41005

[25] G. Plonka. Approximation order provided by refinable function vectors. Constr. Approx., 13:221-244, 1997. MR98c:41023

[26] L. Velho and D. Zorin. 4-8 subdivision. Comp. Aid. Geom. Des., 18(5):397-427, 2001. 
[27] J. Warren and H. Weimer. Subdivision Methods for Geometric Design: A Constructive Approach. Morgan Kaufmann, 2001.

[28] Y. Xue, T. P.-Y. Yu, and T. Duchamp. Hermite subdivision surfaces: Applications of vector refinability to free-form surfaces. In preparation, 2003.

[29] D. Zorin, P. Schröder, and W. Sweldens. Interpolating subdivision for meshes with arbitrary topology. Computer Graphics Proceedings (SIGGRAPH 96), pages 189-192, 1996.

Department of Mathematical and Statistical Sciences, University of Alberta, Edmonton, Alberta, Canada T6G 2G1

E-mail address: bhan@math.ualberta.ca

$U R L:$ http://www.ualberta.ca/ bhan

Department of Mathematical Science, Rensselaer Polytechnic Institute, Troy, New YORK 12180-3590

E-mail address: yut@rpi.edu

URL: http://www.rpi.edu/ yut

Department of Mathematical Science, Rensselaer Polytechnic Institute, Troy, New YoRK 12180-3590

E-mail address: xuey@rpi.edu 